\title{
A scalar nonlocal bifurcation of solitary waves for coupled nonlinear Schrödinger systems
}

\author{
Alan Champneys* and Jianke Yang ${ }^{\dagger}$
}

\begin{abstract}
An explanation is given for previous numerical results which suggest a certain bifurcation of 'vector solitons' from scalar (single-component) solitary waves in coupled nonlinear Schrödinger (NLS) systems. The bifurcation in question is nonlocal in the sense that the vector soliton does not have a small-amplitude component, but instead approaches a solitary wave of one component with two infinitely far-separated waves in the other component. Yet, it is argued that this highly nonlocal event can be predicted from a purely local analysis of the central solitary wave alone. Specifically the linearisation around the central wave should contain asymptotics which grow at precisely the speed of the other-component solitary waves on the two wings. This approximate argument is supported by both a detailed analysis based on matched asymptotic expansions, and numerical experiments on two example systems. The first is the usual coupled NLS system involving an arbitrary ratio between the self-phase and cross-phase modulation terms, and the second is a coupled NLS system with saturable nonlinearity that has recently been demonstrated to support stable multi-peaked solitary waves. The asymptotic analysis further reveals that when the curves which define the proposed criterion for scalar nonlocal bifurcations intersect with boundaries of certain local bifurcations, the nonlocal bifurcation could turn from scalar to non-scalar at the intersection. This phenomenon is observed in the first example. Lastly, we have also selectively tested the linear stability of several solitary waves just born out of scalar nonlocal bifurcations. We found that they are linearly unstable. However, they can lead to stable solitary waves through parameter continuation.
\end{abstract}

Mathematics Subject Classification: 35Q55, 74J35, 37Gxx.

Keywords: coupled NLS equations, solitary waves, nonlocal bifurcations.

\section{Introduction}

Solitary waves play an important role in the solution dynamics of nonlinear evolution equations. If the solitary waves are stable, they often emerge as final states in an initial-value problem. Even if these waves are unstable, the mere existence of such waves has important implications for solution

\footnotetext{
${ }^{*}$ Department of Engineering Mathematics, University of Bristol, Bristol BS8 1TR a.r.champneys@bris.ac.uk

${ }^{\dagger}$ Department of Mathematics and Statistics, The University of Vermont, 16 Colchester Avenue, Burlington, VT 05401, USA jyang@emba.uvm.edu
} 
evolutions. In recent years, it has been discovered that complicated solitary waves could bifurcate from simple solitary waves.

Local bifurcations of 'wave and daughther waves' ('vector solitons') from single-component waves ('scalar solitons') have been studied in various forms of coupled nonlinear Schrödinger (NLS) systems $[4,5,6,10,14,17]$. The bifurcation is local in that bifurcated solitary wave is infinitesimally close to the original solitary wave (as a graph) at the point of bifurcation. The condition for such a local bifurcation to occur is based on the linearisation around the single-component pulse having a solution with purely decaying asymptotics at infinity (see Secs. 2 and 3 below).

Nonlocal bifurcations are where the bifurcated solitary wave is not infinitessimally close to the original wave at the point of bifurcation. These have been reported numerically in the usual coupled NLS equations (the generalised Manakov system, involving an arbitrary ratio between the self-phase and cross-phase studied in Sec. 3 below, henceforth referred to as the CNLS system) $[5,14,15]$, and in second- and third-harmonic generation systems $[6,18,19]$. If the bifurcated wave looks like several vector solitons glued together, that nonlocal bifurcation has also been treated analytically by an asymptotic tail-matching method [15] (see Sec. 4 for application of this method in the context of this paper). General mechanisms have also been identified that lead to such vector non-local bifurcations in classes of coupled NLS systems, such as eigenvalue degeneracy or the existence of a local bifurcation $[15,18,20]$. The linear stability of these multiple-pulsed vector solitons for the CNLS system has been studied in [16], and it has been shown that such states are linearly unstable. However, if the bifurcated wave is glued together by scalar (i.e., single-component) solitary waves, no analysis has been performed to our best knowledge. In this paper, we focus on this scalar nonlocal bifurcation. We will show that it is closely related to local bifurcations, and can be treated on the same footing.

The criterion we propose for a scalar nonlocal bifurcation (in Sec. 2) is that the solution of the linearized equation around the central single-component pulse should have only purely growing asymptotics instead of purely decaying asymptotics at infinity. In the following Sections, we test this criterion against two example systems. The first, in Sections 3, is the CNLS system. The second, in Section 5, is a coupled NLS system with saturable nonlinearity. In both cases, good agreement is obtained between our bifurcation condition and the numerics. Near the bifurcation point, we have also developed a detailed asymptotic analysis based on the above-mentioned tailmatching method, which is performed for the CNLS system in Sec. 4. This analysis produces an explicit formula for the spacing between scalar solitons being pieced together, and this formula agrees well with the numerics. An extension of that analytical theory to more general coupled NLS systems, such as our second example studied in Sec. 5 is straightforward, and will be omitted in this paper. Lastly, we discover in the course of our asymptotic analysis that if the curve which defines our proposed criterion for scalar nonlocal bifurcations intersects with boundaries of certain local bifurcations, the nonlocal bifurcation may turn from scalar to non-scalar at the intersection. This phenomenon indeed occurs in the CNLS system. We have also studied the linear stability of solitary waves just born out of scalar nonlocal bifurcations. The results suggest that these waves are always linearly unstable. However, they can lead to stable solitary waves through parameter continuation in the second model. 


\section{A geometric argument}

Consider a general system of ordinary differential equations (ODEs) of the form

$$
\begin{gathered}
u_{x x}-u+f_{1}(u, v) u=0, \\
v_{x x}-\omega^{2} v+f_{2}(u, v) v=0,
\end{gathered}
$$

where $0<\omega<1$. It is assumed that $f_{1}$ and $f_{2}$ are smooth nonlinear functions of their arguments which vanish as $(u, v) \rightarrow(0,0)$ and may well depend on other system parameters. Moreover they are such that the problem with $v \equiv 0$ or with $v \equiv 0$, which are both invariant subspaces within the four-dimensional phase space of the ODE system $(2.1),(2.2)$, contain even homoclinic solutions

$$
\begin{array}{ll}
(u(x), v(x))=\left(u_{h}(x), 0\right), \quad u_{h}(-x)=u_{h}(x), \quad u \rightarrow 0 \quad \text { as } & x \rightarrow \pm \infty, \\
(u(x), v(x))=\left(0, v_{h}(x)\right), \quad v_{h}(-x)=v_{h}(x), \quad v \rightarrow 0 \quad \text { as } \quad x \rightarrow \pm \infty .
\end{array}
$$

In what follows we shall use the terms 'pulse' 'homoclinic to the origin', 'solitary wave' and 'soliton' entirely synonymously. We shall refer to the invariant subspace homoclinic solutions (2.3) and (2.4) as scalar $u$ - and $v$-pulses respectively. These scalar solitons are contrasted with vector solitons which are homoclinic solutions that have non-zero $u$ and $v$ components. Note that all homoclinic solutions to the origin are generic, that is they persist under parameter perturbation, since the system is both reversible and Hamiltonian [3].

Now consider the linearisation of $(2.1),(2.2)$ around the $u$-pulse

$$
\begin{gathered}
u_{x x}=u+\left\{\frac{\partial}{\partial u} f_{1}\left[u_{h}(x), 0\right] u_{h}(x)+f_{1}\left[u_{h}(x), 0\right]\right\} u, \\
\left.v_{x x}=\omega^{2} v+f_{2}\left[u_{h}(x), 0\right)\right] v .
\end{gathered}
$$

Note that the linear equations decouple. So let us look at the specific class of solutions to this linear problem which have $u=0$. Now we have to simply solve the second equation (2.6). The general asymptotics of such solutions satisfy

$$
v \rightarrow c_{1}^{ \pm} e^{-\omega x}+c_{2}^{ \pm} e^{\omega x}+o\left(e^{-\omega|x|}\right) \text { as }|x| \rightarrow \pm \infty
$$

for some constants $c_{1}^{ \pm}$and $c_{2}^{ \pm}$. Note that we are able to write the $o(\cdot)$-term by the assumption that $0<\omega<1$ so that the asymptotics of $f_{2}(u(x), 0)$ decay more rapidly than $\exp (-\omega|x|)$.

Consider even solutions (2.7) of (2.6), hence $c_{i}^{+}=c_{i}^{-}:=c_{i}, i=1,2$. This defines a unique solution (up to scale) for the linear initial-value problem (2.6) for $x \geq 0$. Now suppose that at a particular value of $\omega$, this solution had a particular tail asymptotics (2.7) with $c_{2}=0$ (see Fig. 1(b)). Then the solution to the linear problem would be localized. Going back to the fully nonlinear problem, by standard bifurcation theory results, we have satisfied the necessary condition for the local bifurcation of a wave and daughter wave consisting of the mother $u$-pulse and a small-amplitude $v$-component. The vanishing of $c_{2}$ is a codimension-one condition, and hence local bifurcations will lie on lines in a parameter plane. As already mentioned in the Introduction, the existence of such bifurcations in coupled NLS systems have been established by a number of authors.

Suppose instead that we find a solution at some parameter value that satisfies $c_{1}=0$. Note that this describes a pure exponentially growing solution to leading order as $x \rightarrow \pm \infty$. See 
Fig. 1(a). However, consider the nonlinear implications of this within the four-dimensional phase space $\left\{\left(u, u^{\prime}, v, v^{\prime}\right): u=u^{\prime}=0\right\}$ of the $\operatorname{ODE}(2.1),(2.2)$. We have found an initial condition for an even solution that is an infinitesimal perturbation of $u_{h}(0)$, and which is attracted as $x \rightarrow \infty$ towards the invariant plane $V:=\left\{\left(u, u^{\prime}, v, v^{\prime}\right): u=u^{\prime}=0\right\}$. Moreover, this rate of attraction is faster $(\sim \exp (x))$ than the exponential contraction or expansion with that plane $(\sim \exp ( \pm \omega x))$ near the origin. Hence the condition that $0<\omega<1$ ensures that the invariant plane $V$ is normally hyperbolic.. Using standard results for normally hyperbolic manifolds, the eventual behaviour of this perturbed trajectory is governed by its behaviour on $V$. The fact $c_{1}=0$ implies that the trajectory is attracted onto the local unstable manifold within $V$. But the local unstable manifold on $V$ is precisely the piece of trajectory that forms the $v$-pulse solution $v_{h}$. Also since we are talking about an infinitesimal perturbation to the underlying pulse $u_{h}$, the time taken to be attracted to $v_{h}$ in this way is arbitrarily long. Hence we have the scalar nonlocal bifurcation of two $v_{h}$ pulses 'at $\pm \infty$ ' as depicted in Fig. 1(a).

The above is only a plausible argument, but it is highly appealing from an intuitive point of view. In Sec. 4, we will develop an in-depth asymptotic theory for this scalar nonlocal bifurcation in the CNLS equations. The results of that theory fully support the above intuitive argument.

Before proceeding to the examples, let us make a few short remarks here.

1. First, note the need to assume that $0<\omega<1$. This was required in order to make the $u=0$ invariant plane normally hyperbolic, or equivalently to assume that the asymptotic attraction onto this plane was $o\left(e^{-\omega x}\right)$. If this condition is violated, then there is no sense in which the perturbed trajectory converges only to the unstable manifold within $V$ and hence the argument fails.

2. Second, it is interesting to note that our analysis suggest that local and scalar nonlocal bifurcations can be treated on an equal footing. One requires that $c_{1}$ vanishes, the other that $c_{2}$ vanishes. It is perfectly possible to imagine a scenario where, as a parameter is varied, the even solution $v(x)$ to the linear problem (2.6) generates extra internal oscillations. If it does so in a smooth way, then it is clear that it must pass repeatedly through successive zeros of $c_{1}$ and $c_{2}$. Hence one would find each scalar nonlocal bifurcation sandwiched between two successive local bifurcations.

3. In fact, in the above it is not quite enough to assume that $c_{1}$ vanishes for a scalar nonlocal bifurcation. We must have that $c_{2}$ has the correct sign to attach to the component of the unstable manifold of the nonlinear equation that contains the pure $v$-pulse. In all the examples below, the pure- $v$ equation is odd and hence both $v_{h}$ and $-v_{h}$ are solutions. Thus either sign of $c_{2}$ will lead to a scalar nonlocal bifurcation.

4. Finally, in systems with odd nonlinearity, the above arguments can be repeated to find antisymmetric scalar nonlocal bifurcations where the two daughter waves at infinity are $v_{h}$ and $-v_{h}$.

We now turn to two examples to test the validity of this approximate reasoning. 


\section{Example 1: the CNLS equations}

The usual coupled nonlinear Schrödinger (CNLS) equations may be written in dimensionless form as

$$
\begin{aligned}
i U_{t}+U_{x x}+\left(|U|^{2}+\beta|V|^{2}\right) U & =0 \\
i V_{t}+V_{x x}+\left(|V|^{2}+\beta|U|^{2}\right) V & =0 .
\end{aligned}
$$

They have been used to describe the interaction between wave packets in dispersive conservative media, and also the interaction between orthogonally polarized components in nonlinear optical fibres; see $[14,5]$ and references therein. Looking for steady solutions of the form

$$
U=e^{i \omega_{1}^{2} t} u(x), \quad V=e^{i \omega_{2}^{2} t} v(x),
$$

and performing scaling so that $\omega_{1}=1$ and $\omega_{2}=\omega$, we arrive at the following set of ODEs

$$
\begin{gathered}
u_{x x}-u+\left(u^{2}+\beta v^{2}\right) u=0, \\
v_{x x}-\omega^{2} v+\left(v^{2}+\beta u^{2}\right) v=0 .
\end{gathered}
$$

Here $\beta$ is a real and positive cross-phase-modulational coefficient, and $\omega(\geq 0)$ is a propagation constant parameter. It is noted that if $[u(x ; \omega), v(x ; \omega]$ is a solution, then another solution at propagation constant $\frac{1}{\omega}$ can be obtained via the transformation [14]

$$
\left[\frac{1}{\omega} v\left(\frac{x}{\omega} ; \omega\right), \frac{1}{\omega} u\left(\frac{x}{\omega} ; \omega\right)\right] .
$$

Thus, in this paper, we restrict $0 \leq \omega \leq 1$.

We note that when $\beta=1$, the partial differential equations (PDEs) (3.1), (3.2) are called the Manakov system which is integrable [8]. In this case, all solitary waves of the ODE system (3.3) and (3.4) have closed-form analytical expressions [1]. When $\beta=0$, the PDEs are two copies of the single NLS equation which is also integrable [21]. The solitary waves for $\beta=0$ are simply sech pulses. When $\beta \neq 0$ or 1 , the structure of solitary waves in this system is much more complicated. This structure was partially unraveled in $[4,2,1,5,14,15]$. It is known that local bifurcations occur along curves in the $(\beta, \omega)$-plane that are given by closed form expressions (see below). These local bifurcations are where wave and daughter-wave structures are born. In other words, at local bifurcations, a small and localized $v$ component develops from a pure $u$-pulse. It was also observed numerically that scalar nonlocal bifurcations such as described in this paper occur. That is, passing through the bifurcation is a single component pulse, for which $v$ is zero everywhere, and $u$ is given by a sech function. Bifurcating from this is a solution for which the $u$ component remains about the same, but the $v$ component suddenly develops two pulses which are far separated from the central $u$ pulse. These $v$ pulses can be symmetrically or antisymmetrically distributed. Their sizes jump from zero to a certain finite size across the bifurcation. In this section, we analytically determine the boundaries of these scalar nonlocal bifurcations through the criterion developed in Sec. 2 and compare them with direct numerical results.

\subsection{Local and scalar nonlocal bifurcations}

First, we recall the results for local bifurcations in this system $[4,17,14]$, assuming that a small $v$-component bifurcates from a pure $u$-pulse. Thus, at the bifurcation point, the $v$ component is 
infinitesimally small. Thus the $u$-component is simply governed by the equation

$$
u_{x x}-u+u^{3}=0
$$

whose homoclinic solution is

$$
u(x)=\sqrt{2} \operatorname{sech} x .
$$

According to standard results, a necessary condition of a local bifurcation of a homoclinic solution with a small-amplitude $v$ component from the $u$-pulse (3.7) is that there is a non-trivial localised solution to the linearised problem of the $v$-component. This takes the form of a linear Schrödinger equation

$$
v_{x x}-\omega^{2} v+2 \beta \operatorname{sech}^{2} x v=0,
$$

and for local bifurcation we require

$$
v \rightarrow 0 \quad \text { as } \quad|x| \rightarrow \pm \infty .
$$

This equation can be solved exactly [7] as we now explain. With the variable transformation

$$
v=\operatorname{sech}^{s} x \psi, \quad \xi=\sinh ^{2} x,
$$

where

$$
s=\frac{\sqrt{1+8 \beta}-1}{2}
$$

the Schrödinger equation (3.8) becomes

$$
\xi(1+\xi) \phi_{x x}+\left[(1-s) \xi+\frac{1}{2}\right] \phi_{x}+\frac{1}{4}\left(s^{2}-\omega^{2}\right) \phi=0,
$$

which is a hyper-geometric equation. Its even and odd solutions are

$$
\begin{gathered}
\phi_{1}=F\left(\frac{1}{2} \omega-\frac{1}{2} s,-\frac{1}{2} \omega-\frac{1}{2} s, \frac{1}{2},-\xi\right), \\
\phi_{2}=\sqrt{\xi} F\left(\frac{1}{2} \omega-\frac{1}{2} s+\frac{1}{2},-\frac{1}{2} \omega-\frac{1}{2} s+\frac{1}{2}, \frac{3}{2},-\xi\right),
\end{gathered}
$$

where $F$ is the hyper-geometric function. In order for the solution $v_{1}=\operatorname{sech}^{s} x \phi_{1}$ to decay to zero as $x$ goes to infinity, one must have

$$
\frac{1}{2} \omega-\frac{1}{2} s=-n_{1},
$$

where $n_{1}$ is a non-negative integer, and $n_{1}<\frac{1}{2} s$. Then the $v_{1}$ solution is

$$
v_{1}=\operatorname{sech}^{s} x \sum_{k=0}^{n_{1}} \frac{\left(-n_{1}\right)_{k}\left(-\frac{1}{2} \omega-\frac{1}{2} s\right)_{k}(-\xi)^{k}}{\left(\frac{1}{2}\right)_{k} k !},
$$

which decays to zero as $\xi^{-\omega / 2}$ (i.e., $\left.e^{-\omega x}\right)$. In the solution $(3.15),(a)_{k}$ is defined as

$$
(a)_{k} \equiv \begin{cases}a(a+1)(a+2) \ldots(a+k-1), & k>0 \\ 1, & k=0 .\end{cases}
$$

In order for solution $v_{2}=\operatorname{sech}^{s} x \phi_{2}$ to decay to zero as $x$ goes to infinity, one must have

$$
\frac{1}{2} \omega-\frac{1}{2} s+\frac{1}{2}=-n_{2},
$$


where $n_{2}$ is a non-negative integer, and $n_{2}<\frac{1}{2}(s-1)$. Then the $v_{2}$ solution is

$$
v_{2}=\operatorname{sech}^{s} x \sinh x \sum_{k=0}^{n_{2}} \frac{\left(-n_{2}\right)_{k}\left(-\frac{1}{2} \omega-\frac{1}{2} s+\frac{1}{2}\right)_{k}(-\xi)^{k}}{\left(\frac{3}{2}\right)_{k} k !},
$$

which also decays to zero as $\xi^{-\omega / 2}$ (i.e., $\left.e^{-\omega x}\right)$.

When conditions (3.14) and (3.17) are combined, we find that the boundaries for local bifurcations are

$$
\omega=\omega_{n}^{L B}(\beta)=s-n,
$$

where $s$ is given by (3.10), $n$ is a non-negative integer, and $n<s$. The first boundary $(n=0)$ exists for any $\beta \geq 0$; the second boundary $(n=1)$ exists only for $\beta \geq 1$; the third boundary $(n=2)$ exists only for $\beta \geq 3$; etc. The first three boundaries $\omega_{0,1,2}^{L B}$ are plotted in Fig. 2 as dashed lines for illustration. Note from the above construction that even $n$ corresponds to the existence of symmetric bifurcating waves (even in both $u$ and $v$ ) whereas odd $n$ corresponds to anti-symmetric bifurcation (even in $u$, odd in $v$ ).

Now, how can we define the scalar nonlocal bifurcations? It can be noted that on the above local bifurcation boundaries, the appropriate solution $v_{1}$ or $v_{2}$ has the following asymptotic behavior:

$$
v(x) \longrightarrow \operatorname{sgn}^{n}(x)\left(c_{n} e^{\omega|x|}+d_{n} e^{-\omega|x|}+o\left(e^{\omega|x|}\right)\right), \quad \text { as } \quad|x| \rightarrow \infty,
$$

where $c_{n}=0$, and $d_{n}$ is a non-zero constant. In other words, this $v$ solution is localized. Condition $c_{n}=0$ in the asymptotics (3.20) is the condition for local bifurcations in this problem.

Now, following the arguments laid out in Sec. 2, a scalar nonlocal bifurcation occurs when the $v$-component of the linearised equation around the $u$-pulse satisfies conditions at infinity that it has a purely growing component. That is, scalar nonlocal bifurcations occur when one of the $v_{1}$ and $v_{2}$ solutions of the Schrödinger equation (3.8) has the following asymptotic behavior:

$$
v(x) \longrightarrow \operatorname{sgn}^{j}(x)\left(\alpha e^{\omega|x|}+\gamma e^{-\omega|x|}+o\left(e^{-\omega|x|}\right)\right) \quad \text { as } \quad|x| \rightarrow \infty,
$$

with $\gamma=0$ but some non-zero $\alpha$, and $j=0$ or 1 is an integer indicating the symmetry of the $v$ solution. Now, a remarkable thing happens. Because it is easy to see using the solutions of Eq. (3.8) obtained above, that condition $\gamma=0$ in the asymptotic equation (3.21) is exactly satisfied on the boundary curves

$$
\omega=\omega_{n}^{N L B}(\beta):=n-s, \quad 0<n-s<1,
$$

where $n$ is a non-negative integer and $s(\beta)$ was defined in Eq. (3.10). In fact, on these boundaries, function $v$ has an unbounded solution

$$
\hat{v}_{1}=\operatorname{sech}^{s} x \sum_{k=0}^{n_{1}} \frac{\left(-n_{1}\right)_{k}\left(\frac{1}{2} \omega-\frac{1}{2} s\right)_{k}(-\xi)^{k}}{\left(\frac{1}{2}\right)_{k} k !}
$$

when $n=2 n_{1}$ is even, and an unbounded solution

$$
\hat{v}_{2}=\operatorname{sech}^{s} x \sinh x \sum_{k=0}^{n_{2}} \frac{\left(-n_{2}\right)_{k}\left(\frac{1}{2} \omega-\frac{1}{2} s+\frac{1}{2}\right)_{k}(-\xi)^{k}}{\left(\frac{3}{2}\right)_{k} k !},
$$


when $n=2 n_{2}+1$ is odd. The asymptotic behaviors of these solutions are

$$
\hat{v}(x) \longrightarrow \operatorname{sgn}^{n}(x)\left(g_{n} e^{\omega|x|}+h_{n} e^{(\omega-2)|x|}\right), \quad|x| \rightarrow \infty,
$$

where

$$
g_{n}= \begin{cases}\frac{2^{s}\left(\frac{1}{2} \omega-\frac{1}{2} s\right)_{n_{1}}}{4^{n}\left(\frac{1}{2}\right)_{n_{1}}}, & n=2 n_{1}, \\ \frac{2^{s-1}\left(\frac{1}{2} \omega-\frac{1}{2} s+\frac{1}{2}\right)_{n_{2}}}{4^{n_{2}}\left(\frac{3}{2}\right)_{n_{2}}}, & n=2 n_{2}+1,\end{cases}
$$

and $h_{n}$ is another constant which can be easily calculated. When $\omega<1$, the second term in (3.25) decays faster than $e^{-\omega|x|}$. Thus the coefficient $\gamma$ in the asymptotics (3.21) is zero on these boundaries.

The boundary curve for scalar nonlocal bifurcations (3.22) can be written alternatively as

$$
\beta=\beta_{n}^{N L B}(\omega)=\frac{1}{8}\left\{(2 n-2 \omega+1)^{2}-1\right\} .
$$

These boundaries are plotted in Fig. 2 as solid lines for comparison with boundaries of local bifurcations (dashed lines), which are given according to (3.19) by

$$
\beta=\beta_{n}^{L B}(\omega)=\frac{1}{8}\left\{(2 n+2 \omega+1)^{2}-1\right\} .
$$

Hence, by construction, the curves of nonlocal bifurcations simply represent the continuation of local bifurcation curves through $\omega=0$ (and mapped back up via $\omega \rightarrow-\omega$, since only $\omega^{2}$ appears in the equations). In particular, at the singular value $\omega=0$ we have that $\omega_{n}^{N L B}=\omega_{n}^{L B}$.

Lastly, we note that the first scalar nonlocal bifurcation curve on the left of Fig. 2 needs a little special treatment. In fact, this solid curve is given by equation

$$
\omega= \begin{cases}-s, & -\frac{1}{8} \leq \beta \leq 0 \text { and } \omega \leq \frac{1}{2} \\ \frac{1}{2}[1+\sqrt{1+8 \beta}], & -\frac{1}{8} \leq \beta \leq 0 \text { and } \omega \geq \frac{1}{2}\end{cases}
$$

In other words, the lower branch of this curve is as given by Eq. (3.22) with $n=0$, but its upper branch is given by a different function. It can be shown that on this upper branch, the solution of the Schrödinger equation (3.8) also has the asymptotics (3.21) with $\gamma=j=0$. This curve is the only scalar nonlocal bifurcation boundary whose functional form is partially different from (3.22).

\subsection{Numerical results}

So we have found curves on which our proposed condition for scalar nonlocal bifurcations derived in Sec. 2 is satisfied. It remains to be seen what happens to the fully nonlinear equations for this example along such curves. In Sec. 4 below we shall consider this problem via asymptotic analysis. In this section we turn to numerical methods.

First let us demonstrate further properties of the structure of solutions to the linearised problem (3.8) by computation of its even and odd solutions as the parameters vary. Figure 3 depicts solutions 
of the constrained linear boundary value problems

$$
v_{x}(0)=0, \quad \int_{0}^{X} v(x)^{2} d x=\text { const. }
$$

and

$$
v(0)=0, \quad \int_{0}^{X} v(x)^{2} d x=\text { const. },
$$

for even and odd solutions respectively. Here $X$ is a large positive constant. At the right-hand boundary point we can distinguish between solution components that decay with exponential rate $e^{-\omega x}$ and those which grow with rate $e^{\omega x}$ by considering the corresponding eigenvectors in the $\left(v, v^{\prime}\right)$-plane. Hence we can define boundary functions

$$
w_{1}=v_{x}(X)+\omega v(X), \quad w_{2}=v_{x}(X)-\omega v(X),
$$

so that a zero of $w_{1}$ defines a solution with no exponentially growing component whereas zeros of $w_{2}$ define solutions with no component that decays like $e^{-\omega x}$. Hence according to the above definitions, $w_{1}=0$ can be used as a numerical test function for local bifurcations and $w_{2}=0$ as a test function for scalar nonlocal bifurcations.

Specifically Fig. 3 depicts the results of a numerical continuation of even and odd solutions to (3.8), satisfying (3.30) and (3.31) respectively, for fixed $\omega$ as $\beta$ is increased from zero. It can be seen that an alternating sequence of zeros of functions $w_{1}$ and $w_{2}$ occurs as $\beta$ increases. At the values of each of the zeros we plot the mode shape $v(x)$. Note that each successive pair of zeros corresponds to the function gaining an extra internal zero. The particular computation was carried out with $X=10$. For this value it was found that the $\beta$-values of the depicted zeros of $w_{1}$ and $w_{2}$ correspond to those of the analytic formulae (3.27) and (3.28) to within 5 decimal places. Increasing $X$ resulted in more accuracy, but an increase in the singularity of the boundary-value problem close to each zero of $w_{1}$.

For this example we have analytic formulae for the conditions defining local and scalar nonlocal bifurcations. Hence these computational results can be interpreted as developing numerical confidence in our method of detecting them in situations where analytic formulae do not exist (as in Sec. 5 below). Also, they provide geometrical insight. Thinking of the phase space $\left(v, v_{x}\right)$, the conditions for local and scalar nonlocal bifurcations are that the solution for large $x$ should lie in one of the two eigendirections. By continuous dependence on initial condition results, then we have that if there are a succession of local bifurcations with increasing number of internal zeros upon increasing a parameter, then the solution at 'time' $X$ must rotate in the phase plane. In so doing, we can not avoid having a scalar nonlocal bifurcation sandwiched between each two successive local bifurcations, see Fig. 4.

Next, let us numerically investigate actual solitary wave bifurcations near the proposed scalar nonlocal bifurcation curve (3.22). First, we consider those curves with $n=1$ and 2 . The scalar nonlocal bifurcations near these curves have been numerically explored in [14]. The results are reproduced in Figs. 5 and 6. In each figure, solitary waves at three different locations of the parameter plane are shown: one is close to the local bifurcation curve (3.19) (dashed line), another one is in the interior, and the third is close to the theoretical scalar nonlocal bifurcation boundary (3.22). Displays of these solitary waves are meant to show the reader how solitary waves continuously deform from wave and daughter wave structures as system parameters $\beta$ and $\omega$ vary. As we can see, in both cases, scalar nonlocal bifurcations indeed occur on the theoretical curves (3.22) (see panel (c) 
in both figures). In addition, the numerical bifurcation boundaries (circles) fall precisely on the theoretical curves. So for these cases, our theory is fully supported by numerics. We have also found similar agreement for the case $n=0$ in the nonlocal bifurcation boundary (3.22).

However, the $n=3$ case is more complicated. The bifurcation for this case is shown in Fig. 7 . The solid line in the parameter plane (upper left panel) is the theoretical curve (3.22) for scalar nonlocal bifurcations. Circles are numerically detected nonlocal bifurcation boundaries. Notice that the numerical boundary falls onto the theoretical curve (3.22) only in the lower part. There the nonlocal bifurcation is indeed scalar, consistent with the geometric argument of Section 2. This can be confirmed in Fig. 7 (e). But in the upper and middle parts, the numerical boundary deviates from the theoretical curve (3.22). The reason turns out to be that, in these parts, the actual nonlocal bifurcation is not scalar. Indeed, an inspection of Fig. 7 (c, d) shows that the bifurcated solitary waves in these parts are not scalar NLS solitons pieced together. Rather, they are true vector solitons pieced together. Thus our analysis for scalar nonlocal bifurcations does not apply here. We note that near the upper part of the numerical boundary, the center of the bifurcated wave is a wave and daughter wave structure with $n=1$ [see Eq. (3.19)], and it is flanked by two single-hump vector solitons on the two sides. This piecing together of different vector solitons as a nonlocal consequence of local bifurcation has been analytically studied in [15] before. It was shown there that the boundary for this type of non-scalar nonlocal bifurcation is precisely the boundary of local bifurcations (3.19) (here with $n=1$ ). This is indeed the case. When the local-bifurcation boundary (3.19) for $n=1$ is plotted as a dashed curve there, it agrees with the numerical boundary (circles) very well. The bifurcation in the middle part of the parameter region is also non-scalar. It is clear from Fig. 7 (d) that this bifurcation is somewhere in between the non-scalar bifurcation of Fig. 7 (c) and the scalar bifurcation of Fig. 7 (e). In fact, it is appropriate to consider this middle part of the bifurcation boundary as a transition between the non-scalar bifurcation in the upper part and the scalar bifurcation in the lower part.

Numerical searching has revealed that there is no scalar nonlocal bifurcation observed along the branch corresponding to (3.22) with $n=3$ above the point at which the non-scalar bifurcations start. Thus the condition (3.22) can be at best a necessary condition for scalar nonlocal bifurcations. Why does the bifurcation deviate from scalar here? and where exactly does this deviation begin? These questions could not be answered by the approximate geometric argument in Sec. 2. However, an answer will be revealed in a matched asymptotic analysis in the next section. We will show that the deviation starts where the curve (3.22) with $n=3$ intersects the local bifurcation boundary $\omega=1 / s$ of $v$-pulses.

\section{Matched asymptotic theory for scalar nonlocal bifurcations in the CNLS equations}

To theoretically explain the scalar nonlocal bifurcation results in the previous section, an analytical theory will now be constructed. This theory has three objectives. The first one is to prove that the boundaries of scalar nonlocal bifurcations are indeed given by the condition that the solution of the linear Schrödinger equation (3.8) has only the purely growing component, i.e., Eq. (3.22). The second objective is to obtain an analytical formula for the spacing between the $v$-pulses and the central $u$-pulse when the parameters are close to the boundary of scalar nonlocal bifurcations. The third objective is to determine when nonlocal bifurcations can deviate from scalar to non- 
scalar. The technique we will use is similar to the tail-matching method as developed in [15] for the construction of multi-pulse trains, but important modifications need to be made. Throughout this analysis, we require $\omega<1$ as above.

Suppose the ODE system (3.3) and (3.4) allows a solution where the $u$-component is symmetric and has a dominant pulse in the center (at $x=0$ ), while the $v$-component is symmetric or antisymmetric and has two dominant pulses on the two sides of the $u$-pulse (at $x= \pm \Delta$ ). Our main assumption is that the $v$-pulses are well-separated from the central $u$-pulse, i.e., $\Delta \gg 1$. Then we can divide the solution into three regions: (I) the left $v$-pulse region centered at $x=-\Delta$; (II) the central $u$-pulse region centered at $x=0$; and (III) the right $v$-pulse region centered at $x=\Delta$. Below, we will determine the solutions in each of these three regions. Note that midway between region II and I or III, both the $u$ and $v$ solutions are very small. Thus they are approximately governed by the linear parts of Eqs. (3.3) and (3.4), hence these solutions are linear combinations of purely exponentially growing and purely exponentially decaying functions to leading order. If these tail asymptotics from two adjacent regions can match each other, then a solitary wave can be found. This is the essence of the tail-matching method.

When the $v$-component is symmetric or antisymmetric, the tail-matching treatment between regions II and III becomes the same as that between regions II and I. Thus, we will focus only on matching between regions I and II.

In region I, the solution can be written as

$$
u=\tilde{u}_{I}, \quad v=v_{0}+\tilde{v}_{I}
$$

where

$$
v_{0}=\sqrt{2} \omega \operatorname{sech}[\omega(x+\Delta)], \quad \tilde{u}_{I} \ll 1, \quad \tilde{v}_{I} \ll 1
$$

(see Figs. 5(c) and 6(c)). In new coordinates

$$
\xi=x+\Delta
$$

the small $\tilde{u}_{I}$ component satisfies the linear Schrödinger equation

$$
\tilde{u}_{I \xi \xi}-\tilde{u}_{I}+\beta v_{0}^{2}(\xi) \tilde{u}_{I}=0
$$

to leading order. To obtain solitary waves, we demand that

$$
\tilde{u}_{I}(\xi) \longrightarrow 0, \quad \xi \rightarrow-\infty .
$$

At large positive $\xi$ values, this $\tilde{u}_{I}$ solution must match the tails of the dominant $u$ solution

$$
u_{0}(x)=\sqrt{2} \operatorname{sech} x
$$

in region II. This matching dictates that the asymptotic behavior of $\tilde{u}_{I}$ at large $\xi$ values is

$$
\tilde{u}_{I}(\xi) \longrightarrow 2 \sqrt{2} e^{-\Delta} \cdot e^{\xi}, \quad \Delta \gg \xi \gg 1 .
$$

The linear equation (4.4), together with the boundary conditions (4.5) and (4.7), completely determines the $\tilde{u}_{I}$ solution in region $\mathrm{I}$. 
Now we determine the small $\tilde{v}_{I}$ component in region I. When Eq. (4.1) is substituted into (3.4), and terms of order $\tilde{v}_{I}^{2}, \tilde{v}_{I}^{3}$ and $\tilde{u}_{I}^{2} \tilde{v}_{I}$ dropped, we find that, to leading order, $\tilde{v}_{I}$ satisfies the following equation

$$
\tilde{v}_{I \xi \xi}-\omega^{2} \tilde{v}_{I}+3 v_{0}^{2}(\xi) \tilde{v}_{I}=-\beta \tilde{u}_{I}^{2} v_{0}(\xi) .
$$

We note that it is important to retain the inhomogeneous term in Eq. (4.8), as otherwise, that equation with the vanishing boundary condition at negative infinity would always produce a localized solution which is impossible to match to the $v$ solution in region II. The boundary conditions for solution $\tilde{v}_{I}$ are

$$
\tilde{v}_{I} \longrightarrow 0, \quad \xi \rightarrow-\infty
$$

and

$$
\tilde{v}_{I} \longrightarrow \alpha e^{\omega \xi}+\gamma e^{-\omega \xi}-\frac{4 \sqrt{2} \beta \omega e^{-2 \Delta}}{1-\omega} e^{(2-\omega) \xi}, \quad \Delta \gg \xi \gg 1,
$$

where $\alpha$ and $\gamma$ are constants. The last term in Eq. (4.10) is contributed from the inhomogeneous term of Eq. (4.8). In deriving it, the asymptotic behaviors of $\tilde{u}_{I}$ and $v_{0}$ solutions were used [see Eqs. (4.2) and (4.7)].

Next, we determine the solutions in region II. In this region, the solutions can be written as

$$
u=u_{0}+\tilde{u}_{I I}, \quad v=\tilde{v}_{I I},
$$

where $u_{0}(x)$ is given in Eq. (4.6), and $\tilde{u}_{I I}, \tilde{v}_{I I} \ll 1$ (see Figs. 5(c) and 6(c)). Here we only need to focus on the $\tilde{v}_{I I}$ solution. This solution satisfies the equation

$$
\tilde{v}_{I I x x}-\omega^{2} \tilde{v}_{I I}+\beta u_{0}^{2}(x) \tilde{v}_{I I}=0
$$

to leading order. The leading asymptotic behavior of this solution at $x \ll-1$ is

$$
\tilde{v}_{I I} \longrightarrow \bar{\gamma}\left(e^{-\omega x}+\delta e^{\omega x}\right), \quad-\Delta \ll x \ll-1,
$$

where $\bar{\gamma}$ and $\delta$ are constants. If we only consider solitary waves with symmetric or antisymmetric $v$ components, then the above $\tilde{v}_{I I}$ solution would have the same symmetry. This symmetry condition would uniquely determine the coefficient $\delta$. The constant $\bar{\gamma}$ is selected by the condition that the tail asymptotics of $\tilde{v}_{I I}$ solution at $x \ll-1$ in region II must match the $v$ solution (4.1) at $\xi \gg 1$ in region I. This matching gives $\bar{\gamma}$ as $=(2 \sqrt{2} \omega+\gamma) e^{-\omega \Delta}$. Recall that $\tilde{v}_{I} \ll 1$, and thus $\gamma \ll 1$. As a result, to the leading order,

$$
\bar{\gamma}=2 \sqrt{2} \omega e^{-\omega \Delta}
$$

This matching also gives the relation

$$
\alpha=\bar{\gamma} \delta e^{-\omega \Delta}=2 \sqrt{2} \omega \delta e^{-2 \omega \Delta} .
$$

One may wonder why the third term in the $\tilde{v}_{I}$ asymptotics $(4.10)$ is not matched by $\tilde{v}_{I I}$ asymptotics (4.13). In fact, there is a smaller term in the $\tilde{v}_{I I}$ solution which is proportional to $e^{(2-\omega) x}$. This term arises due to the product of $u_{0}^{2}$ and the $e^{-\omega x}$ component in the leading $\tilde{v}_{I I}$ solution (4.13) [see Eq. (4.12)]. One can check that this term will exactly match the third term in the $\tilde{v}_{I}$ asymptotics (4.10). So there is no contradiction here. But this is a minor issue which is not critical to our analysis.

Now we are in a position to derive a formula for the spacing $\Delta$ between the $v$-pulses and the middle $u$-pulse. This formula comes from the solvability condition for the $\tilde{v}_{I}$ equation (4.8) together with 
the boundary conditions (4.9), (4.10) and (4.15). It is noted that Eq. (4.8) is self-adjoint, and it has a localized homogeneous solution $v_{0 \xi}$ due to the spatial translation invariance of the ODE equation (3.4). Calculating the integrals of products between $v_{0 \xi}$ and the two sides of Eq. (4.8) from $-\infty$ to $y$, and integrating by parts, we get

$$
\int_{-\infty}^{y}-\beta \tilde{u}_{I}^{2} v_{0} v_{0 \xi} d \xi=\left.\left(\tilde{v}_{I \xi} v_{0 \xi}-\tilde{v}_{I} v_{0 \xi \xi}\right)\right|_{-\infty} ^{y} .
$$

When $\Delta \gg y \gg 1$, substituting the boundary conditions (4.9), (4.10) and (4.15) into the above equation, we find that

$$
\int_{-\infty}^{y}-\beta \tilde{u}_{I}^{2} v_{0} v_{0 \xi} d \xi \longrightarrow-16 \delta \omega^{4} e^{-2 \omega \Delta}+\frac{32 \beta \omega^{3} e^{-2 \Delta}}{1-\omega} e^{2(1-\omega) y}, \quad y \rightarrow \infty .
$$

The above equation is the leading two-term expansion for the integral on its left-hand side. When $y$ approaches infinity, this integral diverges. But we can separate this divergent part from the rest of the integral. Notice that

$$
\frac{32 \beta \omega^{3} e^{-2 \Delta}}{1-\omega} e^{2(1-\omega) y}=\int_{-\infty}^{y} 64 \beta \omega^{3} e^{-2 \Delta} e^{2(1-\omega) \xi} d \xi
$$

Thus Eq. (4.17) can be rewritten as

$$
\beta \int_{-\infty}^{\infty}\left[\tilde{u}_{I}^{2}\left(v_{0}^{2}\right)_{\xi}+128 \omega^{3} e^{-2 \Delta} e^{2(1-\omega) \xi}\right] d \xi=32 \delta \omega^{4} e^{-2 \omega \Delta}
$$

The integral above is no longer divergent. In fact, one can use the asymptotic relations (4.5) and (4.7) to check that the integrand in that integral approaches zero exponentially as $|x|$ goes to infinity.

Eq. (4.19) gives a formula for spacing $\Delta$ when the system parameters $\beta$ and $\omega$ are specified. This formula can actually be made more explicit as follows. Recall that function $\tilde{u}_{I}$ is determined by Eq. (4.4) and boundary conditions (4.5) and (4.7). Under the notation

$$
\tilde{u}_{I}(\xi)=2 \sqrt{2} e^{-\Delta} \phi(\xi)
$$

function $\phi(\xi)$ is then uniquely specified by the following equation and boundary conditions

$$
\begin{gathered}
\phi_{\xi \xi}-\phi+\beta v_{0}^{2}(\xi) \phi=0, \\
\phi(\xi) \longrightarrow \begin{cases}0, & \xi \rightarrow-\infty, \\
e^{\xi}, & \xi \rightarrow \infty,\end{cases}
\end{gathered}
$$

where $v_{0}(\xi)$ is given by Eqs. (4.2) and (4.3). Under these notations, formula (4.19) simplifies as

$$
e^{-2(1-\omega) \Delta}=\frac{4 \omega^{4} \delta}{\beta I}
$$

where $I$ is the integral

$$
I=\int_{-\infty}^{\infty}\left[\phi^{2}\left(v_{0}^{2}\right)_{\xi}+16 \omega^{3} e^{2(1-\omega) \xi}\right] d \xi
$$

Recall that the constant $\delta$ is defined by Eqs. (4.12) and (4.13). To be more explicit, $\delta$ is defined by

$$
\psi_{x x}-\omega^{2} \psi+\beta u_{0}^{2}(x) \psi=0,
$$


and

$$
\psi(x) \longrightarrow e^{-\omega x}+\delta e^{\omega x}, \quad x \rightarrow-\infty .
$$

In other words, $\delta$ is the coefficient of the purely decaying component of the Schrödinger equation (4.25) at $x=-\infty$. Function $\psi$ 's boundary condition at $x=\infty$ is provided by the symmetry of the $v$ component in the solitary wave we are seeking. Since we are focusing on symmetric and antisymmetric $v$ components, function $\psi$ would have the same symmetry. This symmetry helps to uniquely determine the $\delta$ coefficient in the above linear problem.

Formula (4.23) is the key result of this section. It explicitly gives the expression for the spacing $\Delta$ in solitary waves bifurcating from scalar nonlocal bifurcations. Several observations quickly follow from this formula. First, solitary waves from scalar nonlocal bifurcations exist only when parameters $\delta$ and $I$ have the same sign. Second, when $\delta=0, \Delta$ goes to infinity. Thus this is a boundary of scalar nonlocal bifurcations. This condition is precisely the one proposed in Sec. 2 . For the coupled NLS system, $\delta=0$ on the curves (3.22). One may notice from formula (4.23) that $\Delta$ also goes to infinity when $I=\infty$. However, $I=\infty$ does not correspond to a boundary of scalar nonlocal bifurcations. The reason is as follows. For the coupled NLS system, $I=\infty$ on the local bifurcation boundaries of pure $v$-pulses:

$$
\omega=\frac{1}{s-n},
$$

where $n$ is an integer and $0 \leq n<s$. This is because on these boundaries, the solution $\phi$ of Eq. (4.21) which satisfies the zero boundary condition at $\xi=-\infty$ [see Eq. (4.22)] is always localized. Thus in order for it to satisfy the boundary condition (4.22) at $\xi=\infty, \phi$ must be infinitely large. Hence $I=\infty$. The above fact applies to the solution $\tilde{u}_{I}$ of Eq. (4.4) as well: on the local bifurcation boundary (4.27), solution $\tilde{u}_{I}$ satisfying boundary conditions (4.5) and (4.7) is infinitely large. When this happens, our original assumption $\tilde{u}_{I} \ll 1$ for scalar nonlocal bifurcations breaks down. Hence if there is a nonlocal bifurcation here at all, it would not be scalar: the pulses on the two wings would be true vector solitons. Thus $I=\infty$ does not give a boundary of scalar nonlocal bifurcations. Consequently, a scalar nonlocal bifurcation boundary is given entirely by the condition $\delta=0$, which is our previous condition. An interesting and subtle issue is: what if curves $\delta=0$ and $I=\infty$ intersect? As we have discussed above, when $I=\infty$, the nonlocal bifurcation (if there is one) becomes non-scalar. Thus at the locus of $I=\infty$ and $\delta=0$, the nonlocal bifurcation could turn from scalar to non-scalar. Then the bifurcation boundary would deviate from the scalar bifurcation curve $\delta=0$ at the intersection. This phenomenon could, and does, happen. In fact, Fig. 7 gives a good example. Let us reproduce Fig. 7's scalar bifurcation curve [i.e., (3.22) with $n=3$ ] and the true bifurcation boundary in Fig. 8 below (solid line and circles). On top of it, we plot the local bifurcation curve (4.27) of $v$-pulses with $n=0$ (dashed line). We see that the intersection between these two curves is precisely where bifurcation turns from scalar to non-scalar, thus deviation between the numerical bifurcation boundary and the scalar bifurcation boundary starts there. This example and the matched asymptotic analysis above tell us that the previous condition for scalar nonlocal bifurcations is a necessary but not sufficient condition. When local bifurcation curves of $v$-pulses intersect with these necessary-condition curves, non-scalar bifurcations could start, thus scalar bifurcations on part of the necessary-condition curves will not materialize.

The analytical expressions for $\delta$ and $I$ may be possible to obtain, as the linear Schrödinger equations (4.21) and (4.25) can be solved using hyper-geometric functions (see [7] and Sec. 3). But such expressions would be very complex. For practical purposes, it is preferable to determine them numerically. For illustration purposes, we select $\omega=0.6$, and show the numerical values of these 
quantities in Fig. 9 at various $\beta$ values ranging from -1 to 7 . In Fig. 9a, the integral $I$ is shown. This integral is independent of the symmetry of the $v$ solution. In Fig. 9(b, c), the coefficients $\delta$ for anti-symmetric and symmetric $v$ solutions are shown respectively. This figure is helpful in revealing on which side of the scalar nonlocal bifurcation boundary solitary waves can be expected. For instance, at $\omega=0.6, \delta=0$ when $\beta=0.28$ and the $v(x)$ component is anti-symmetric. On the left-hand side of this $\beta$ value, $\delta>0$ and $I>0$, thus solitary waves with symmetric $u$ component and anti-symmetric $v$ component can be expected. But on the right-hand side of this $\beta$ value, $\delta<0$ and $I>0$, thus no such waves can be found. These predictions completely agree with the numerics shown in the previous section. Similar agreement is found near other scalar nonlocal bifurcation curves as well.

As $\omega \rightarrow \omega_{n}^{N L B}(\beta), \delta \rightarrow 0$, thus $\Delta \rightarrow \infty$ according to formula (4.23). Below, we derive the asymptotic formula for $\Delta$ when $\omega \rightarrow \omega_{n}^{N L B}(\beta)$, or equivalently, $\beta \rightarrow \beta_{n}^{N L B}(\omega)$ [see Eq. (3.27)]. The latter limit will be adopted in the following derivation as it is a little more convenient.

When $\beta \rightarrow \beta_{n}^{N L B}(\omega)$, the integral $I$ approaches a finite constant value $I\left(\omega, \beta_{n}^{N L B}\right)$, while $\delta$ goes to zero. Obviously, the asymptotic formula for $\Delta$ crucially depends on the asymptotic formula of $\delta$. We determine $\delta$ 's asymptotic formula by regular perturbation methods below. Equation (4.25) can be rewritten as

$$
\psi_{x x}-\omega^{2} \psi+\beta_{n}^{N L B} u_{0}^{2} \psi=-\epsilon u_{0}^{2} \psi,
$$

where $\epsilon=\beta-\beta_{n}^{N L B}$. When $\epsilon=0$, this equation has an unbounded solution

$$
\psi_{n 0}(x)= \begin{cases}\frac{1}{g_{n}} \hat{v}_{1}(x), & n=2 n_{1} \\ -\frac{1}{g_{n}} \hat{v}_{2}(x), & n=2 n_{2}+1\end{cases}
$$

where functions $\hat{v}_{1,2}(x)$ and constant $g_{n}$ are given by Eqs. (3.23), (3.24) and (3.26). The asymptotic behavior of this solution is that

$$
\psi_{n 0}(x) \longrightarrow e^{-\omega x}+o\left(e^{\omega x}\right), \quad x \rightarrow-\infty
$$

[see Eqs. (3.25)]. When $\epsilon$ is small, solution $\psi$ can be expanded into a regular perturbation series

$$
\psi(x, \beta, \omega)=\psi_{n 0}\left(x, \beta_{n}^{N L B}, \omega\right)+\epsilon \psi_{n 1}\left(x, \beta_{n}^{N L B}, \omega\right)+O\left(\epsilon^{2}\right) .
$$

When this expansion is substituted into Eq. (4.25), at order $\epsilon$, we find that function $\psi_{n 1}$ satisfies the equation

$$
\psi_{n 1 x x}-\omega^{2} \psi_{n 1}+\beta_{n}^{N L B} u_{0}^{2} \psi_{n 1}=-u_{0}^{2} \psi_{n 0} .
$$

Recalling that the boundary conditions for $\psi(x)$ and $\psi_{n 0}(x)$ are given by Eqs. (4.26) and (4.30), thus the boundary condition for function $\psi_{n 1}$ is

$$
\psi_{n 1}(x) \longrightarrow \frac{\delta}{\epsilon} e^{\omega x}, \quad x \rightarrow-\infty .
$$

The linear operator on the left hand side of Eq. (4.32) is self-adjoint. In addition, $\psi_{n 0}(x)$ is a homogeneous solution. Calculating the inner product between $\psi_{n 0}$ and the inhomogeneous term of Eq. (4.32), we readily find that

$$
\int_{-\infty}^{\infty} u_{0}^{2} \psi_{n 0}^{2} d x=\left.\left(\psi_{n 0 x} \psi_{n 1}-\psi_{n 0} \psi_{n 1 x}\right)\right|_{-\infty} ^{\infty}
$$


Substituting the boundary conditions (4.30) and (4.33) into the above relation, we find that for both $\psi(x)$ symmetric and anti-symmetric cases, the constant $\delta$ is given by the asymptotic formula

$$
\delta=\frac{\beta-\beta_{n}^{N L B}}{4 \omega} \int_{-\infty}^{\infty} u_{0}^{2} \psi_{n 0}^{2} d x+O\left(\left(\beta-\beta_{n}^{N L B}\right)^{2}\right) .
$$

We have compared this formula with the numerical values of $\delta$ as displayed in Fig. 9(b,c). The slope $\int_{-\infty}^{\infty} u_{0}^{2} \psi_{n 0}^{2} d x / 4 \omega$ predicted by this formula is in excellent agreement with the numerical slope at $\beta=\beta_{n}^{N L B}$. When this formula is substituted into Eq. (4.23), we finally obtain the leading two-term asymptotic expansion for spacing function $\Delta$ as

$$
\Delta=\frac{1}{2(\omega-1)}\left\{\ln \left(\beta-\beta_{n}^{N L B}\right)+\ln K+O\left(\beta-\beta_{n}^{N L B}\right)\right\},
$$

where the constant $K$ is

$$
K=K_{n}(\omega)=\frac{\omega^{3}}{\beta_{n}^{N L B} I\left(\omega, \beta_{n}^{N L B}\right)} \int_{-\infty}^{\infty} u_{0}^{2} \psi_{n 0}^{2} d x .
$$

Next, we make quantitative comparisons between the spacing formulas (4.23), its leading two-term expansion (4.36) and numerics near the scalar nonlocal bifurcation boundaries (3.22) with $n=1$ and 2 at $\omega=0.6$ and various $\beta$ values (see Fig. 2 ). We remind the reader that $\omega=1-s$ is a boundary for anti-symmetric $v$ components, and $\omega=2-s$ is a boundary for symmetric $v$ components. At $\omega=0.6$, the boundary point is at $\beta_{1}^{N L B}=0.28$ for the former case, and is at $\beta_{2}^{N L B}=1.68$ for the latter case. The analytical spacings from formula (4.23) and its two-term asymptotic expansion (4.36) are shown as dashed and dash-dotted lines in Fig. 10(a, b) for these two cases respectively We have also numerically determined the spacings between the $v$-pulses and the central $u$-pulse in the exact solitary waves. These numerical values are shown as solid lines in Fig. 10(a, b). We see that, when the separation $\Delta$ is large, the formula (4.23) and its asymptotic form (4.36) agree with the numerical values perfectly.

\section{Example 2: Saturable nonlinearity}

As a second example we take the coupled nonlinear Schrödinger system studied by Ostrovskaya and Kivshar [10]

$$
\begin{aligned}
& i U_{t}+U_{x x}+U \frac{|U|^{2}+|V|^{2}}{1+s\left(|U|^{2}+|V|^{2}\right)}=0 \\
& i V_{t}+V_{x x}+V \frac{|U|^{2}+|V|^{2}}{1+s\left(|U|^{2}+|V|^{2}\right)}=0 .
\end{aligned}
$$

This dimensionless model arises after scaling of a model for the incoherent interaction between two linearly polarized optical beams in a biased photorefractive medium. Here $s(0<s<1)$ is an

effective saturation parameter, representing the photorefractive effects. This system is significant because it was shown in [11] that multi-humped stationary pulses may be stable solutions, a result that explains the experimental observations of [9]. In the limit $s \rightarrow 0$, this system reduces to the Manakov equations. 
The solitons in this system are of the form

$$
U(x, t)=e^{i t} u(x), \quad V(x, t)=e^{i \omega^{2} t} v(x),
$$

where $u$ and $v$ are real functions satisfying the following ODEs:

$$
\begin{gathered}
u_{x x}-u+u \frac{u^{2}+v^{2}}{1+s\left(u^{2}+v^{2}\right)}=0, \\
v_{x x}-\omega^{2} v+v \frac{u^{2}+v^{2}}{1+s\left(u^{2}+v^{2}\right)}=0 .
\end{gathered}
$$

Looking for single-component pulses $(u=0$, or $v=0)$ we obtain simple planar equations, which can be shown by phase plane techniques to possess symmetric homoclinic orbits $\pm u_{h}, \pm v_{h}$. Unlike the previous example, we know of no closed form expressions for these solutions other than at $s=0$. So we turn straight away to numerical methods. Once again we restrict to $0<\omega<1$ and look for local and scalar nonlocal bifurcations from the pulse $u_{h}$. Figure 11 shows the analogue of Fig. 2 for this example where the bifurcation boundaries were obtained by numerically imposing the boundary conditions for local bifurcation and for scalar nonlocal bifurcation on the linearised equation

$$
v_{x x}-\omega^{2} v+\frac{u_{h}^{2}(x) v}{1+s u_{h}^{2}(x)}=0 .
$$

Figure 12 depicts the corresponding graphs of $w_{1}(s)$ and $w_{2}(s)$ for fixed $\omega=0.5$, where $w_{1}$ and $w_{2}$ were defined in (3.32). Note that we have qualitatively the same structure as in Fig. 3 in that the mode shapes $v(x)$ gain increasingly many internal zeros as the parameter increases to some limit. Moreover, between each pair of local bifurcations of a given symmetry type there is a scalar nonlocal bifurcation, and vice-versa. Here it would seem that the limit $s \rightarrow 1$ plays the same qualitative role as $\beta \rightarrow \infty$ in the previous model, and we conjecture that there are infinitely many local and scalar nonlocal bifurcations as we approach this limit. This is bourn out by the results in Fig. 11 which show that the curves in the $(s, \omega)$-plane defined by zeros of $w_{1}=0$ and $w_{2}=0$ become increasingly steep as $s \rightarrow 1$. It would be fair to conjecture from the numerics that all curves (apart from the first symmetric scalar nonlocal bifurcation) approach the point $(\omega, s)=(1,1)$. There are some numerical difficulties in computing the nonlocal bifurcation curves up to this point, because the pulse $u_{h}$ becomes infinitely broad as $s \rightarrow 1$, and hence the solution $v(x)$ of the linearised problem which grows exponentially at infinity must be continually rescaled to avoid the solution becoming arbitrarily large.

Now the presence of local bifurcations has been found before in this system; see Fig. 1 in [10] where local bifurcations are found via zeros of a certain integral as a function of $\omega$ for several different values of $s$. Here we have presented a simple procedure for automatically detecting these local bifurcations as a function of all system parameters. As far as we know these curves of local bifurcations are not given by closed form analytic expressions as they were in the previous example.

The question remains whether the curves which have been putatively called scalar nonlocal bifurcations really represent that for the full equations. Figure 13 depicts numerical solutions of the fully coupled nonlinear equations for fixed $\omega=0.5$ in a parameter region between the second local and first scalar nonlocal symmetric bifurcations. A one-parameter family of vector solitons is computed which are born at the local bifurcation. It is found that the solution branch terminates at the value $s \approx 0.8055$ which agrees to four decimal places with the scalar nonlocal bifurcation 
boundary determined from our geometric argument of Sec. 2. Inspection of solitary waves close to this boundary indicates that a scalar nonlocal bifurcation indeed occurs here. Note from Fig. 13(c) panel that the separation varies logarithmicly with the variation of the parameter away from the nonlocal bifurcation boundary, as in the first example [see Eq. (4.36)].

Finally, Fig. 14 indicates the results of a series of similar one-parameter continuations (for either fixed $s$ or fixed $\omega$ ) starting from the same curve of local bifurcations. Observe the agreement between the termination of these branches and the theoretical scalar nonlocal bifurcation boundary. Indeed, in each run the final orbit was found to be a pure $u$-pulse flanked symmetrically by two $v$-pulses, qualitatively the same as in Fig. 13. Similar results starting from each of the local bifurcation boundaries computed in Fig. 11 have found that the bifurcated branches all continue smoothly up to the theoretical scalar nonlocal bifurcation curves. Thus, in this second example, the proposed condition for scalar nonlocal bifurcations and numerics show complete and global agreement. We note that no deviation of the sort in Fig. 7 of the first example occurs here because scalar nonlocal bifurcation boundaries do not intersect with local bifurcation boundaries of $v$-pulses now. In fact, no local bifurcations of daughter $u$-solutions from pure $v$-pulses occur for $s<1$. This is because, unlike in the CNLS example where the transformation $\omega \rightarrow 1 / \omega$ mapped back curves into the domain of interest $(0<\beta<\infty)$, here this transformation takes curves into the region $s>1$.

\section{Linear instability of solitary waves arising from nonlocal bifur- cations}

An important question about solitary waves generated by scalar nonlocal bifurcations is their linear stability. These waves are multi-humped by construction. The common wisdom is that multihump solitary waves in conservative systems are linearly unstable (for instance, see [16]). However, this is not always the case, as stable multihump solitary waves in the saturable coupled NLS model (5.1), (5.2) have been reported [11]. A comprehensive stability analysis of these solitary waves is quite involved and lies outside the scope of the present article. However, in this section, we will selectively test the linear stability of a few such solitary waves as discussed above. These results are suggestive of the linear stability behavior of solitary waves born out of scalar nonlocal bifurcations as a whole.

Our strategy for testing the linear stability is by numerically simulating the linearized equations of the two models $(3.1,3.2)$ and $(5.1,5.2)$ expanded around solitary waves. For both models, we perturb solitary waves as

$$
U(x, t)=e^{i t}\{u(x)+\epsilon \psi(x, t)\}, \quad V(x, t)=e^{i \omega^{2} t}\{v(x)+\epsilon \phi(x, t)\},
$$

where $(u, v)$ is a solitary wave, $\epsilon \ll 1$, and $(\psi, \phi)$ are perturbation functions. Substituting this perturbed solution into each model and dropping terms which are order $\epsilon^{2}$ and higher, the linearized equations for $\psi$ and $\phi$ will be obtained. Then we simulate this linearized system for a long time, starting with a random noise. If the solutions $\psi$ and $\phi$ exponentially grow, then the solitary wave $(u, v)$ is linearly unstable.

First, we consider the coupled NLS model $(3.1,3.2)$. Its nonlocal bifurcation boundary is shown in Fig. 2. At $(\beta, \omega)=(0.29,0.6)$ which is close to the nonlocal bifurcation boundary with $n=1$, the solitary wave born out of this nonlocal bifurcation is displayed in Fig. 15(a) [see also Fig. 5]. 
Numerical simulation of the linearized equation around this solitary wave shows that there is a purely exponentially-growing unstable eigenmode. The unstable eigenfunction is displayed in Fig. 15(b). The unstable eigenvalue is 0.083 (purely real). Thus, this solitary wave is linearly unstable. At $(\beta, \omega)=(1.6,0.6)$ which is close to the nonlocal bifurcation boundary with $n=2$ (see Fig. 2), another solitary wave is born and displayed in Fig. 15(c). We have found that this wave is also linearly unstable. The unstable eigenfunction is shown in Fig. 15(d), and the unstable eigenvalue is 0.13 (purely real).

Next, we consider the saturable model $(5.1,5.2)$. As shown in Fig. 13, at $(s, \omega)=(0.81,0.5)$, there is a solitary wave born out of a nonlocal bifurcation. This solitary wave is reproduced in Fig. 16(a). By simulating the linearized system, we have found that this solitary wave is linearly unstable as well. The unstable eigenfunction is shown in Fig. 16(b), and the unstable eigenvalue is 0.090 (purely real).

From these selective numerical testings, we have reason to believe that solitary waves which are just born out of scalar nonlocal bifurcations in the two models (3.1, 3.2) and (5.1, 5.2) are linearly unstable in general. This belief is consistent with a previously established fact that solitary waves born out of vector nonlocal bifurcations are linearly unstable [16]. However, when these solitary waves have moved far away from the boundaries of nonlocal bifurcations, instability may disappear, and the solitary waves may become stable. In fact, we have found that in the saturable model (5.1, 5.2), when solitary waves of Fig. 13(a) move to the local bifurcation boundary $s=s^{L B}$, they indeed become linearly stable (a similar phenomenon has been reported in [11]). But in the CNLS model $(3.1,3.2)$, solitary waves remain unstable when they move to the boundaries of local bifurcations $[13,12]$.

\section{Discussion}

In this paper we have described by numerical computation, plausible argument and detailed asymptotic analysis a new kind of bifurcation of solitary waves for coupled nonlinear Schrödinger systems. Our geometric argument suggests that this so-called scalar nonlocal bifurcation occurs when the linearization of the nonlinear system around a scalar pulse has purely growing asymptotics at infinity. Our matched asymptotic analysis confirms this condition. It further reveals that when this scalar nonlocal bifurcation boundary intersects with certain local-bifurcation boundaries, the actual nonlocal bifurcation can turn from scalar to non-scalar at the intersection. All these theoretical results are fully supported by our numerics on two coupled NLS systems (3.3), (3.4) and (5.4), (5.5). Our direct numerical simulations suggest that solitary waves which are just born from scalar nonlocal bifurcations are linearly unstable, but they can lead to stable solitary waves by parameter continuation.

It remains an open problem to fully unfold the codimension-two nonlocal bifurcation that occurs when the scalar nonlocal bifurcation boundary intersects the local-bifurcation boundary. In particular it remains unknown whether a scalar non-local bifurcation continues to occur on the upper portion of the solid curve in Fig. 8 (upper left panel). We have found that the wave that starts from the local bifurcation with $n=3$ does not end there, but this does not exclude the possibility of other branches of vector solitons terminating on this possible nonlocal bifurcation boundary. Preliminary numerical searching has not revealed any candidate branches, but a more careful study 
is required.

Finally we should mention that the ideas in this paper were originally motivated by the observation of what was termed a 'jump' bifurcation in [6]. There, a nonlocal bifurcation occurs where a pure- $v$ pulse bifurcates at infinity from a vector soliton. The model in question is for a third-harmonic generation system where the symmetry is such that pure $u$ solutions do not exist. At present it is not clear whether a simple criterion like the one in this paper can be applied. One could conjecture that the jump bifurcation occurs when linearisation around the central vector soliton has precisely the growing exponential asymptotics in its tail of the pure- $v$ pulse. But this conjecture requires careful numerical and analytical confirmations.

\section{Acknowledgments}

The authors acknowledge insightful conversations with Alice Yew and Boris Malomed. This work was initiated while JY was visiting the UK under an EPSRC grant. ARC also holds an Advanced Research Fellowship from the EPSRC. JY's work was also supported by the US National Science Foundation grant DMS-9971712 and The Air Force Office of Scientific Research contract USAF F49620-99-1-0174.

\section{References}

[1] N. N. Akhmediev, A. V. Buryak, J. M. Soto-Crespo, and D. R. Andersen. Phase-locked stationary soliton states in birefringent nonlinear optical fibers. J. Opt. Soc. Am. B, 12:434, 1995.

[2] N.N. Akhmediev and A. Ankiewicz. Novel soliton states and bifurcation phenomena in nonlinear fiber couplers. Physical Review Letters, 70:2395-2398, 1993.

[3] R.L. Devaney. Reversible diffeomorphisms and flows. Trans. Amer. Math. Soc., 218:89-113, 1976.

[4] V.M. Eleonsky, V.G. Korolev, N.E. Kulagin, and L.P. Shil'nikov. Bifurcations of the trajectories at the saddle level in Hamiltonian systems generated by two coupled Schrödinger equations. Chaos, 2:571-579, 1992.

[5] M. Haelterman and A.P. Sheppard. Bifurcation phenomena and multiple soliton bound states in isotropic Kerr media. Phys. Rev. E, 49:3376-3381, 1994.

[6] K. Kolossovski, A.V. Buryak, V.V Steblina, A.R. Champneys, and R.A. Sammut. Higherorder nonlinear modes and bifurcation phenomena due to degenerate parameteric four-wave mixing. Phys. Rev. E, 62:4309-4317, 2000.

[7] L.D. Landau and E.M. Lifshitz. Quantum Mechanics: non-relativistic thoery. Pergamon Press, Oxford, 3rd edition, 1977.

[8] S.V. Manakov. On the theory of two-dimensional stationary self-focusing of electromagnetic waves. Zh. Eksp. Teor. Fiz., 65:1392, 1973. 
[9] M. Mitchell, M. Segev, and D.N. Christodoulides. Observation of multihump multimode solitons. Phys. Rev. Lett., 80:4657, 1998.

[10] E.A. Ostrovskaya and Yu.S. Kivshar. Multi-hump optical solitons in a saturable medium. J. Opt. B: Quantum Semiclass. Opt., 1:77-83, 1999.

[11] E.A. Ostrovskaya, Yu.S. Kivshar, D.V. Skryabin, and W.J Firth. Stability of multi-hump optical solitons. Phys. Rev. Lett., 83:296-299, 1999.

[12] D.E. Pelinovsky. Matrix stability theory for incoherent optical solitons. Preprint.

[13] D.E. Pelinovsky and J. Yang. Internal oscillations and radiation damping of vector solitons. Stud. Appl. Math., 105:245, 2000.

[14] J. Yang. Classification of the solitary wave in coupled nonlinear Schrödinger equations. Physica D, 108:92-112, 1997.

[15] J. Yang. Multiple permanent-wave trains in nonlinear systems. Stud. Appl. Math., 100:127, 1998.

[16] J. Yang. Interactions of vector solitons. Phys. Rev. E, 64:026607, 2001.

[17] J. Yang and D.J. Benney. Some properties of nonlinear wave systems. Stud. Appl. Math., 96:111-139, 1996.

[18] A.C. Yew. Multipulses of nonlinearly coupled schrodinger equations. J. Diff. Eqs., 173:92-137, 2001.

[19] A.C. Yew, A.R. Champneys, and P.J. McKenna. Localised solutions of a coupled first and second harmonic nonlinear shrödinger system. J. Nonlin. Sci., 9, 1999.

[20] A.C. Yew, B. Sandstede, and Jones C.K.R.T. Instability of multiple pulses in coupled nonlinear schrodinger equations. Phys. Rev. E, 61:5886-5892, 2000.

[21] V.E. Zakharov and A.B. Shabat. Exact theory of two-dimensional self-focusing and onedimensional self-modulation of waves in nonlinear media. Zh. Eksp. Teor. Fiz., 61:118, 1971. 
(a)
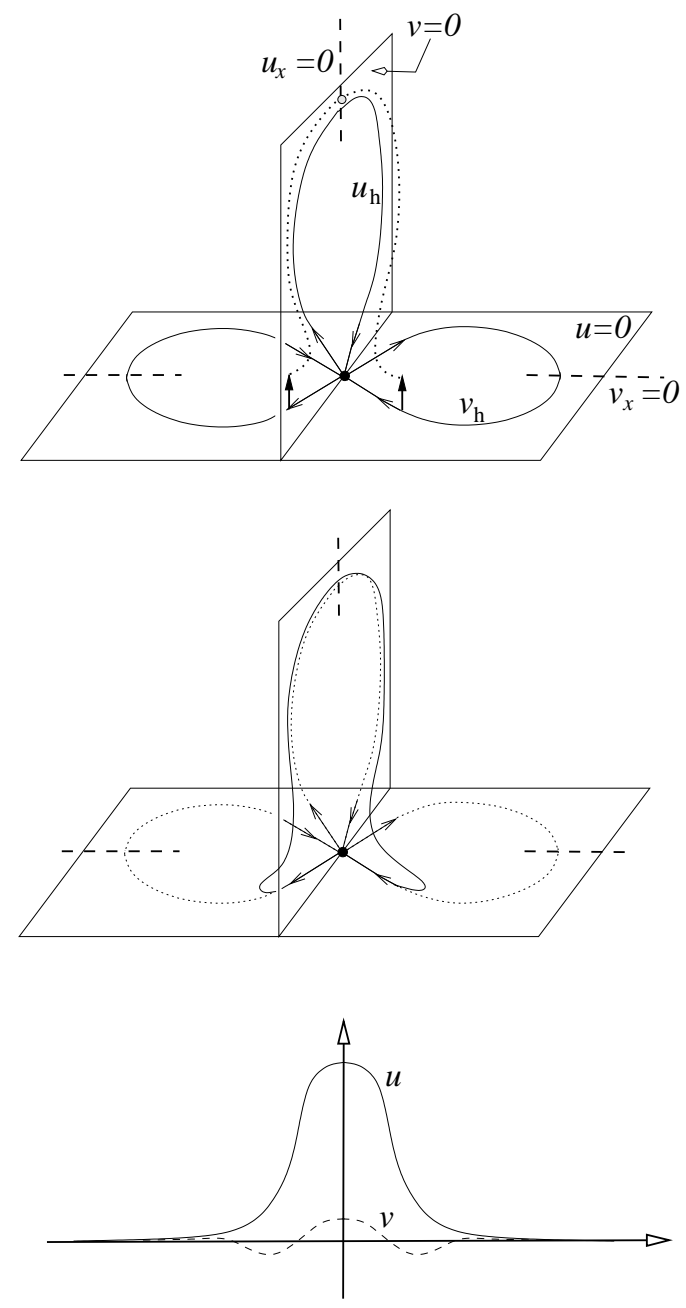

(b)
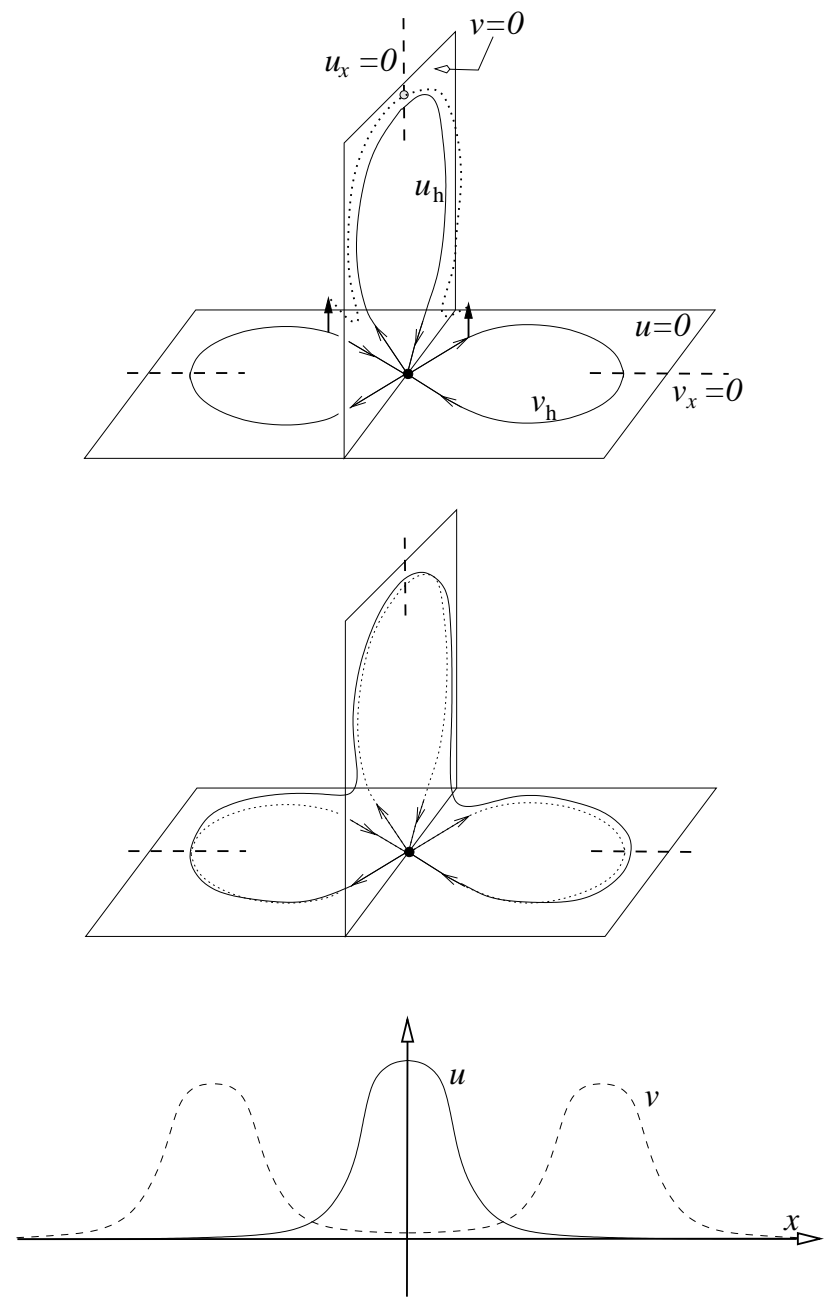

Figure 1: Sketch figure defining (a) scalar nonlocal and (b) local bifurcations. In each case the top panel depicts schematically the invariant planes $\{u \equiv 0\}$ and $\{v \equiv 0\}$ and the solution of the linearised problem around $u_{h}$. The lower two panels depict the corresponding bifurcated vector solitons in phase space and as graphs. 


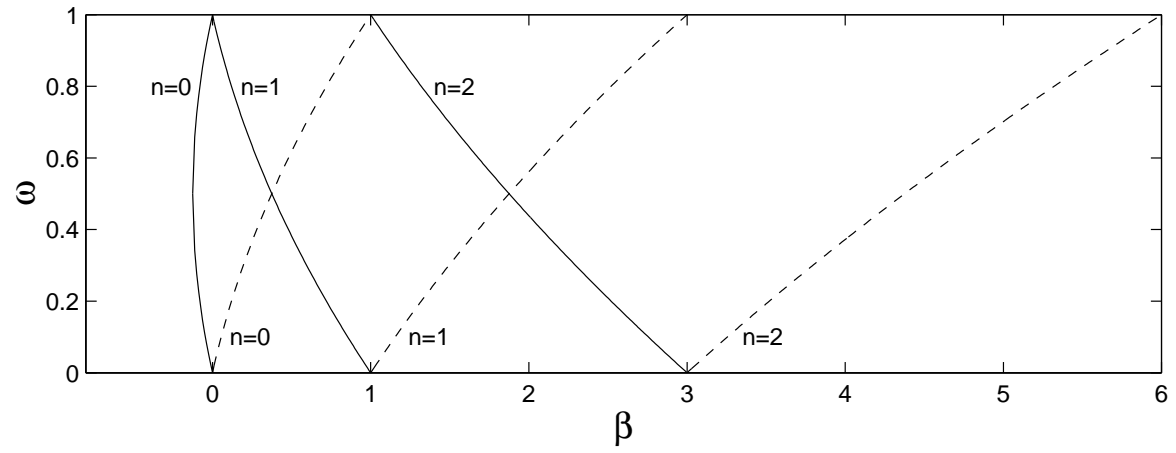

Figure 2: Local and scalar nonlocal bifurcation boundaries in the coupled NLS system (3.3) and (3.4). Scalar nonlocal bifurcation boundaries are solid lines, and are given by Eq. (3.29) [with $n=0$ ] and (3.22) [with $n=1,2$ ]. Local bifurcation boundaries are dashed lines, and are given by Eq. (3.19). 


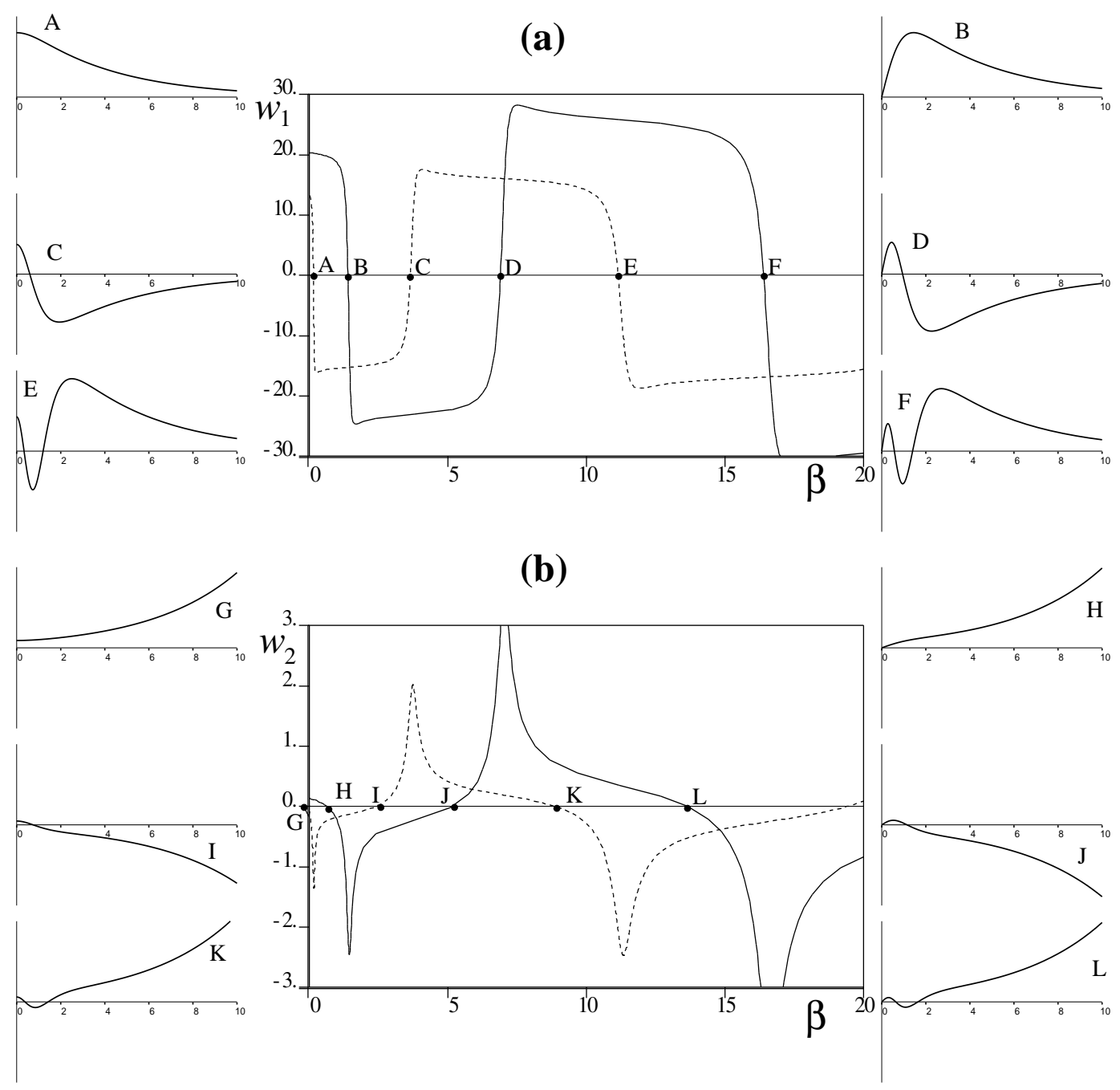

Figure 3: Curves of (a) $w_{1}(\beta)$ and (b) $w_{2}(\beta)$ for the linearisation (3.8) of the CNLS example, computed for $\omega=0.5$ using and interval $x \in[0,10]$. Solid lines represent asymmetric solutions and dashed lines symmetric ones. The inserts depict solutions of the linearised equations at the first three zeros of $w_{1}$, both symmetric and antisymmetric, which define local bifurcations; and of $w_{2}$ which define the necessary conditions for scalar nonlocal bifurcations according to our geometric theory. 




(c) $\beta=\beta_{3}$



(b) $\beta=\beta_{2}$

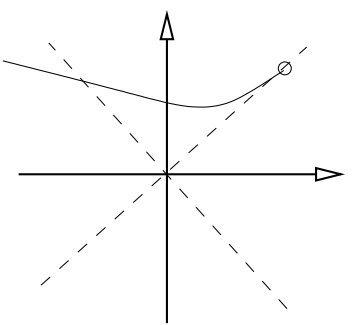

(d) $\left(v(X), v_{x}(X)\right)$

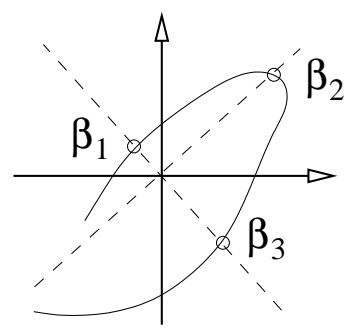

Figure 4: Sketch figure illustrating the large- $x$ asymptotics of even solutions to (3.8) as the parameter $\beta$ varies for fixed $0<\omega<1$. The solution is depicted to the boundary value problem (3.30) up to $x=X$. Between two $\beta$-values (a),(c) at which condition $w_{1}=0$ for local bifurcations occur, there is a $\beta$-value (b) for which $w_{2}=0$. Panel (d) sketches the locus of boundary points $\left(v(X), v_{x}(X)\right.$ as a function of $\beta$. 
(a)
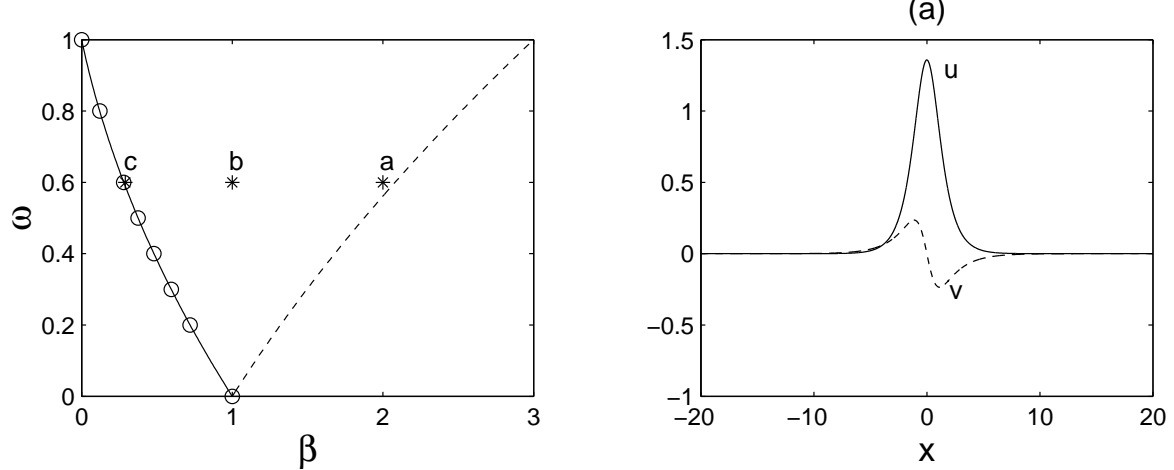

(b)

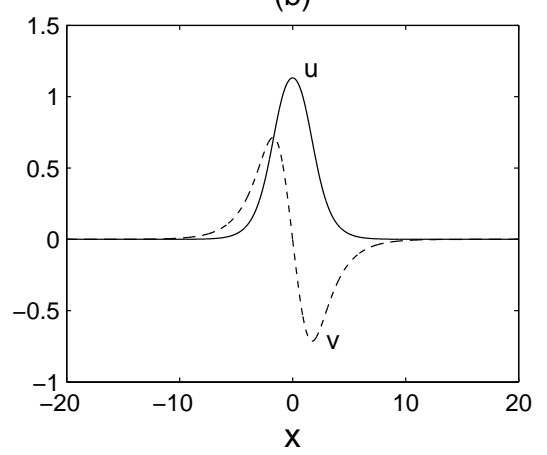

(c)

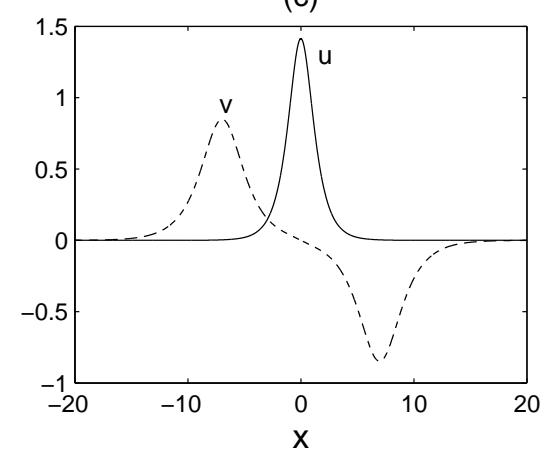

Figure 5: Solitary waves in the parameter region (upper left figure) bounded by the scalar nonlocal bifurcation boundary (3.22) (solid) and local bifurcation boundary (3.19) (dashed) with $n=1$. Circles represent the numerical scalar nonlocal bifurcation boundary for $n=1$ obtained in [14]. Solitary waves at stars marked by letters 'a, b, c' in the parameter region are shown with corresponding letters in the title. 
(a)

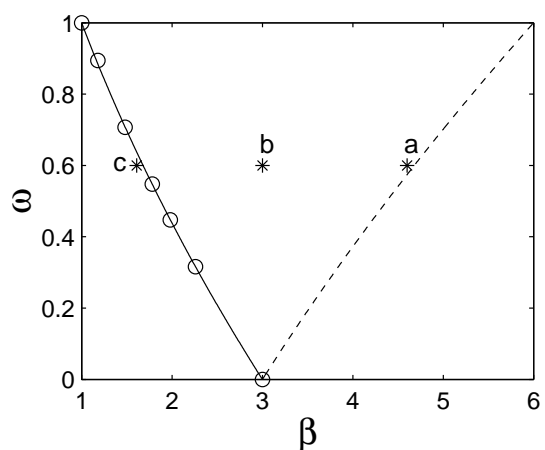

(b)

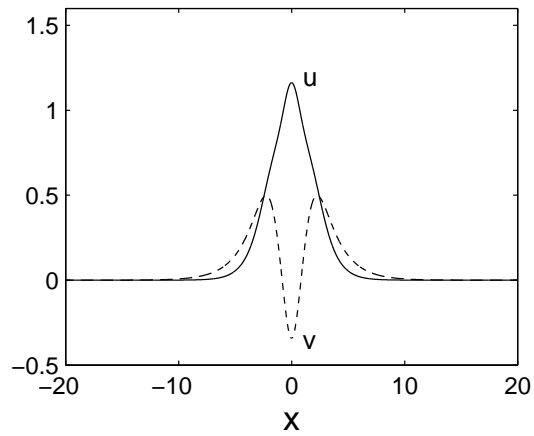

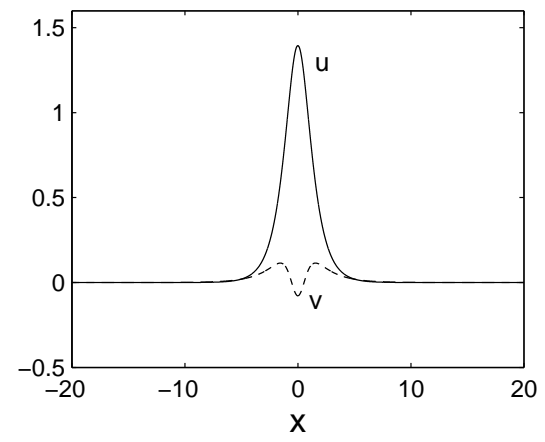

(c)



Figure 6: Solitary waves in the parameter region bounded by the scalar nonlocal bifurcation boundary (3.22) (solid) and local bifurcation boundary (3.19) (dashed) with $n=2$. Circles represent the numerical scalar nonlocal bifurcation boundary for $n=2$ obtained in [14]. 


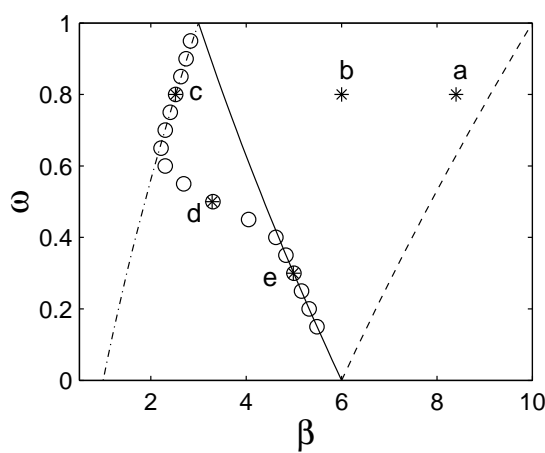

(b)



(d)

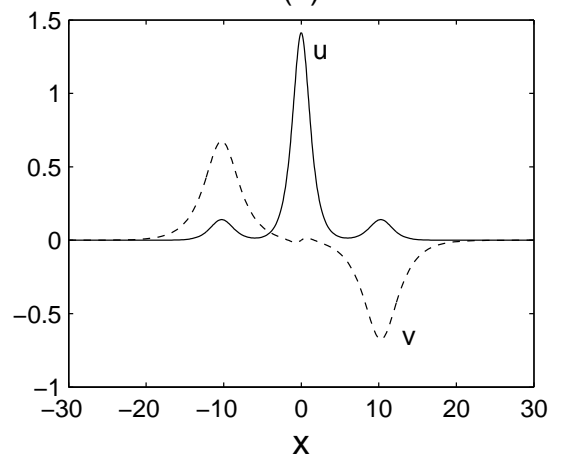

(a)

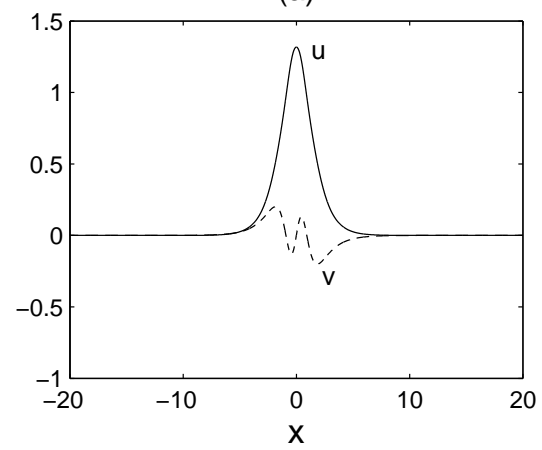

(c)

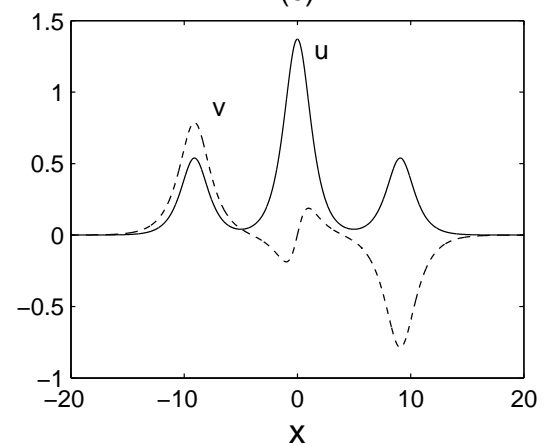

(e)

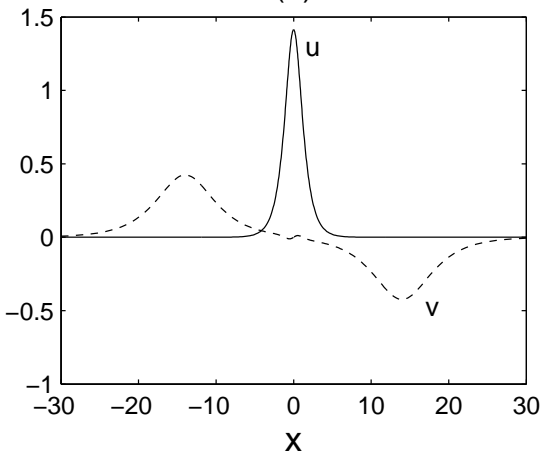

Figure 7: Different types of nonlocal bifurcations appearing near the scalar nonlocal bifurcation curve (3.22) with $n=3$ (solid line) in the coupled NLS system (3.3) and (3.4). Circles are numerically obtained nonlocal bifurcation boundaries. The lower part of the numerical curve lies on the solid line (3.22), and the bifurcation there is indeed scaler [see (e)]. The upper part of the numerical curve lies on the dash-dotted local bifurcation boundary (3.19) with $n=1$, and the nonlocal bifurcation there is non-scalar [see (c)]. The nonlocal bifurcation in the middle part of the numerical curve is somewhere in between scaler and non-scaler [see (d)]. The dashed line in the upper left panel is the local bifurcation curve (3.19) with $n=3$. Stars are parameter values where the solitary waves are shown. 


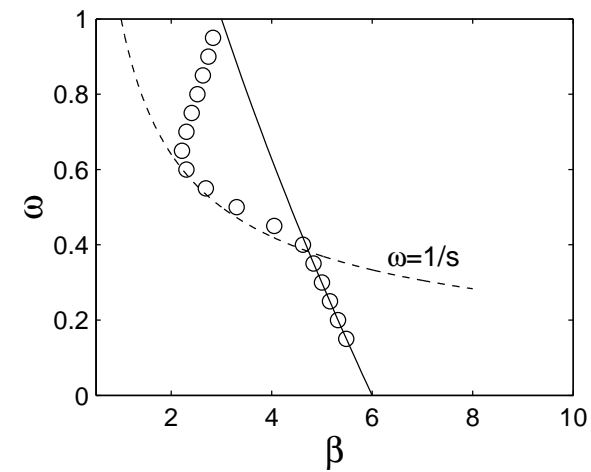

Figure 8: Intersection between the dashed curve $\omega=1 / s$ [see Eq. (3.10)] defining local bifurcations of daughter- $u$ solutions from $v$-pulses (with $n=0$ ) and the solid curve defining the necessary condition (3.22) for scalar nonlocal bifurcations (with $n=3$ ). Circles show the numerical results for nonlocal bifurcations reproduced from Fig. 7.

(a)

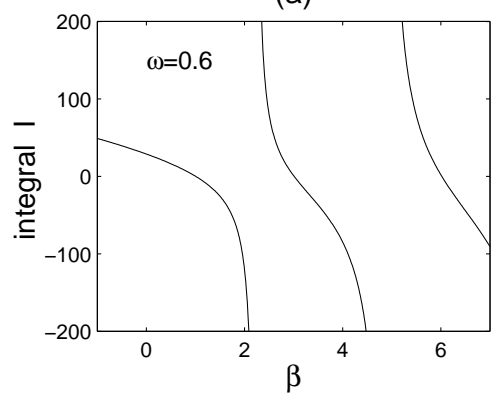

(b)

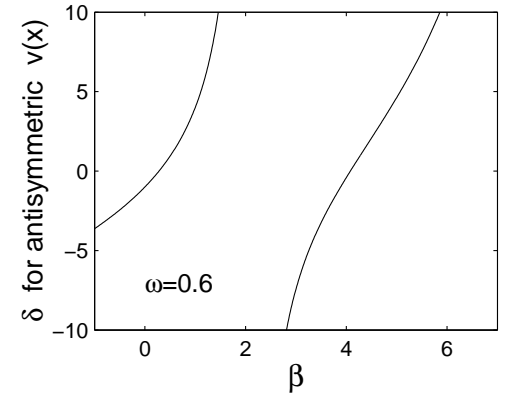

(c)

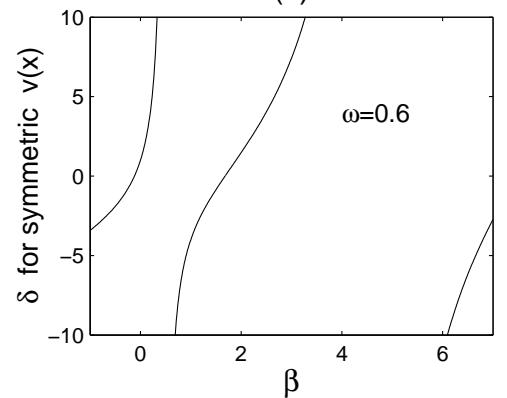

Figure 9: Parameter values $\delta$ and $I$ at $\omega=0.6$ and various $\beta$ values. (a) the integral $I$ values; (b) coefficient $\delta$ for anti-symmetric $v$ components; (c) $\delta$ for symmetric $v$ components. 
(a)

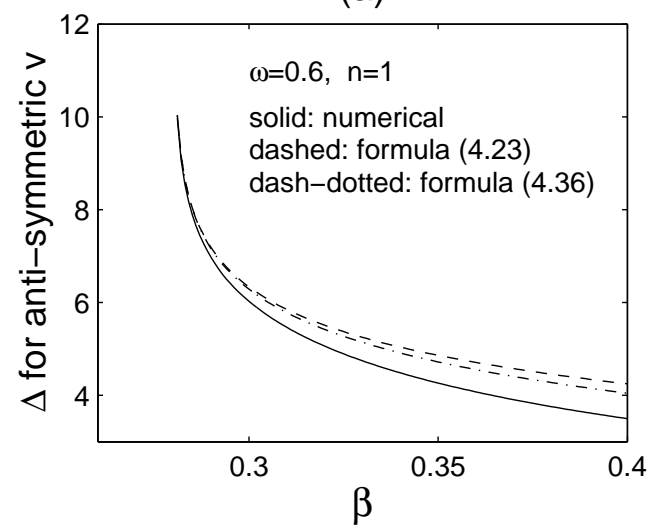

(b)

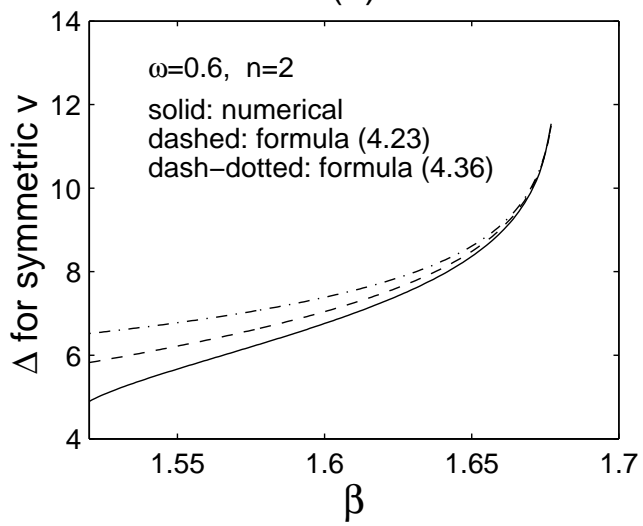

Figure 10: Comparison between the analytical formula (4.23), its leading two-term asymptotic expansion (4.36) and numerical values for spacing $\Delta$ at $\omega=0.6$ and various $\beta$ values. (a) comparison near the scalar nonlocal bifurcation boundary (3.22) with $n=1$; (b) comparison near the scalar nonlocal bifurcation boundary (3.22) with $n=2$.

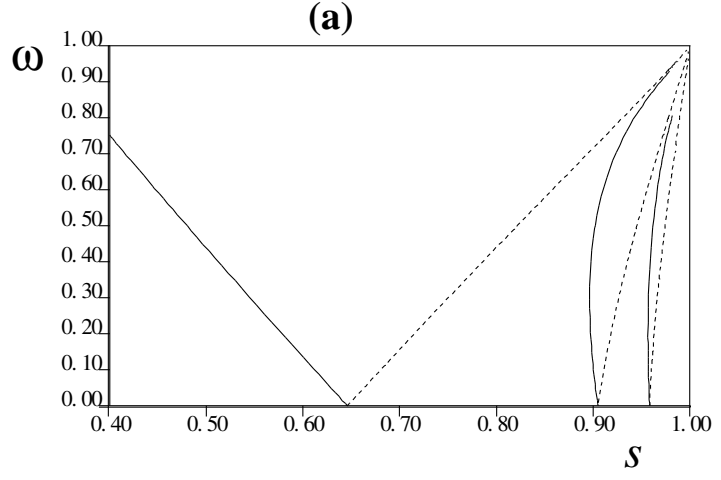

(b)

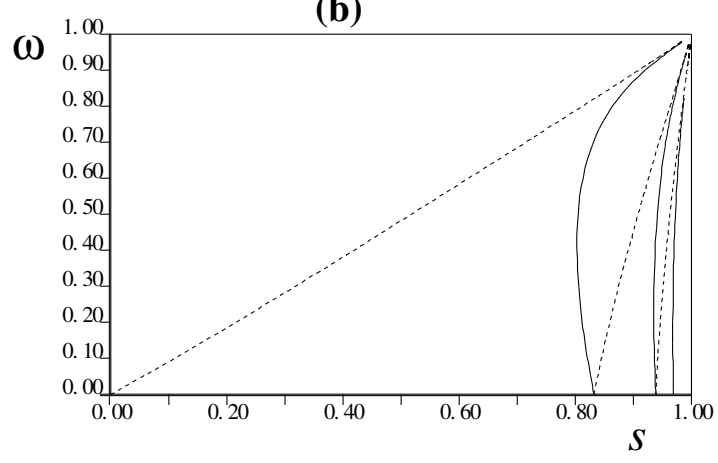

Figure 11: Curves of local (dashed) and scalar nonlocal (solid) bifurcations from the pure $u$-pulse $u_{h}$ of (5.4), (5.5) obtained by numerically solving the linearised equation (5.6) and requiring the solution to have purely decaying or growing asymptotics at infinity. (a) the $v$-component is even; (b) the $v$-component is odd. Only the first three curves of each type is presented. 
(a)

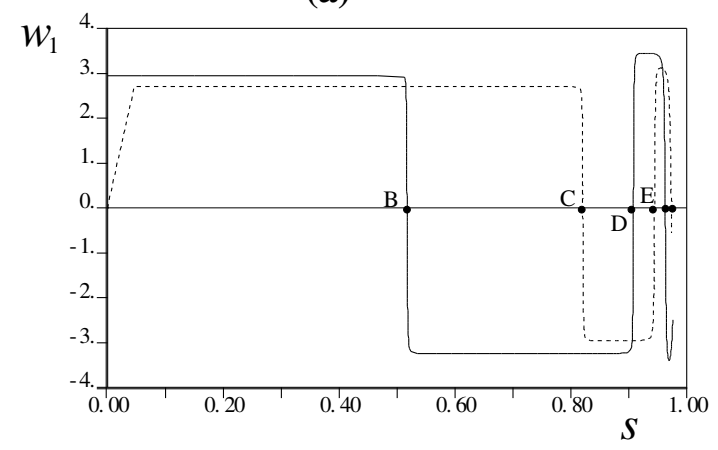

(b)

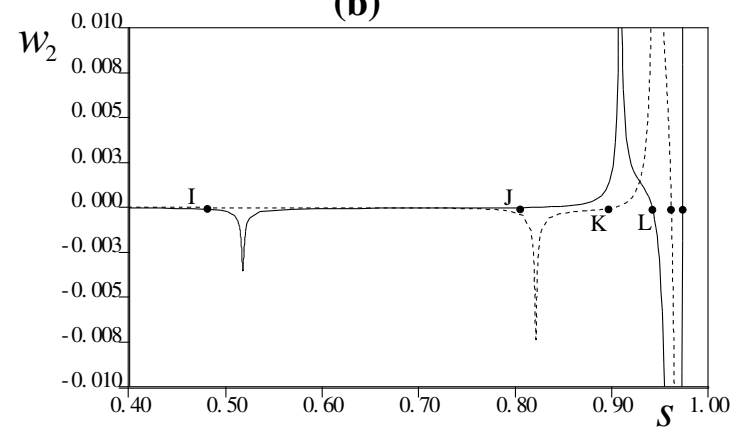

Figure 12: Curves of (a) $w_{1}(s)$ and (b) $w_{2}(s)$ defining respectively local and scalar nonlocal bifurcations according to the theory, for the model (5.4), (5.5) at $\omega=0.5$. Dashed lines correspond to modes with even symmetry and solid lines to odd-symmetric modes. The labeled zeros of each function correspond to mode shapes for $v(x)$ that are qualitatively similar (i.e. having the same structure of zeros) as the corresponding panels of Fig. 3. 

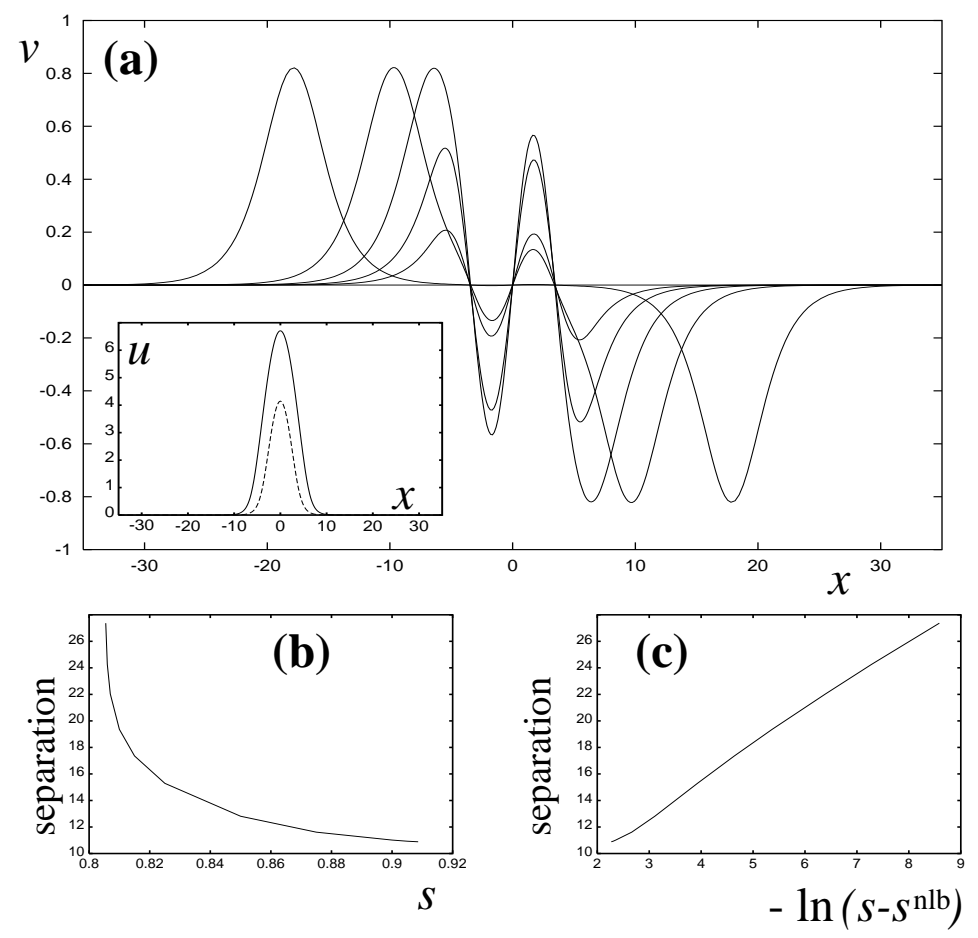

Figure 13: (a) The $v$-component of vector solitons in (5.4), (5.5) for $\omega=0.5$ and $s=0.9075(\approx$ $\left.s^{L B}\right), 0.9,0.85,0.81,0.8055\left(\approx s^{N L B}\right)$. The inset depicts the $u$-component of the vector soliton at $s^{L B}$ (larger peak) and $s^{N L B}$ (smaller peak). In (b) the separation between the two maxima of $|v|$ is plotted as a function of $s$, and (c) plots the same data on a logarithmic scale where the more precise value $s^{N L B}=0.8053134$ is used.

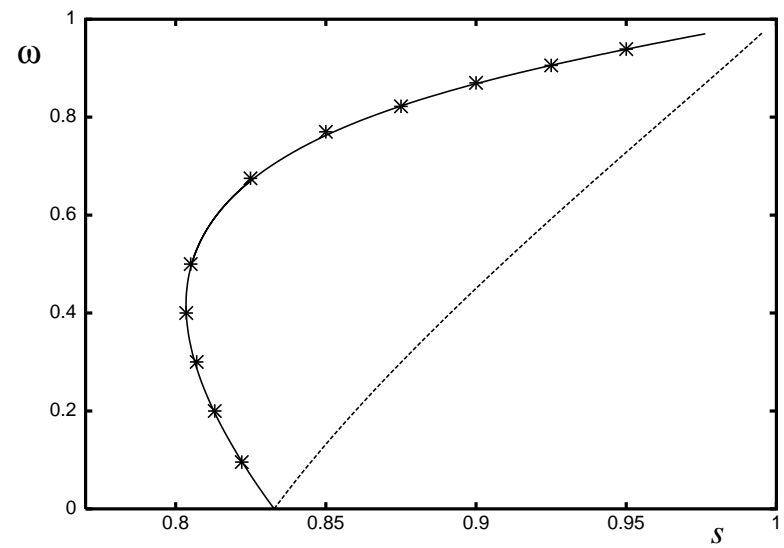

Figure 14: A scalar nonlocal bifurcation boundary for the system (5.4), (5.5). The stars represent scalar nonlocal bifurcation boundaries that were obtained by solving the full nonlinear equations. The solid line was obtained from the condition $w_{2}=0$ for the linearised equation (5.6). The dashed line is a similarly obtained curve of local bifurcations from which these particular vector solitons are born. 
(a)

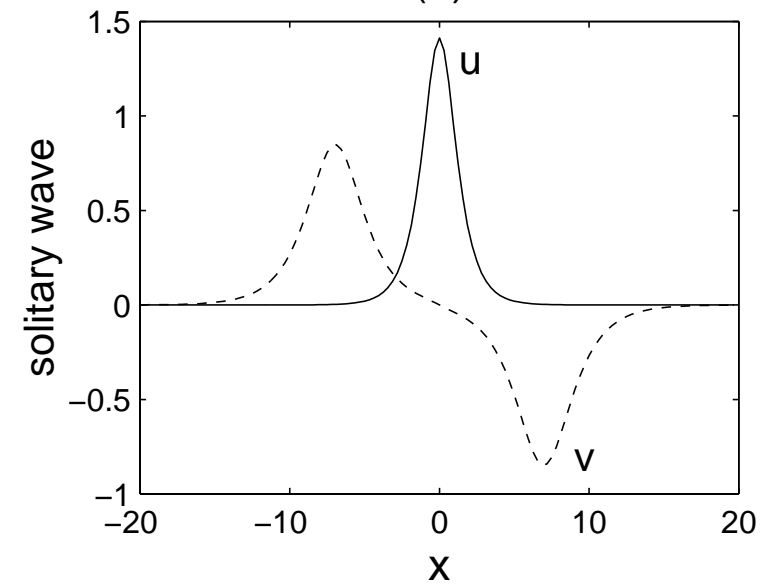

(c)

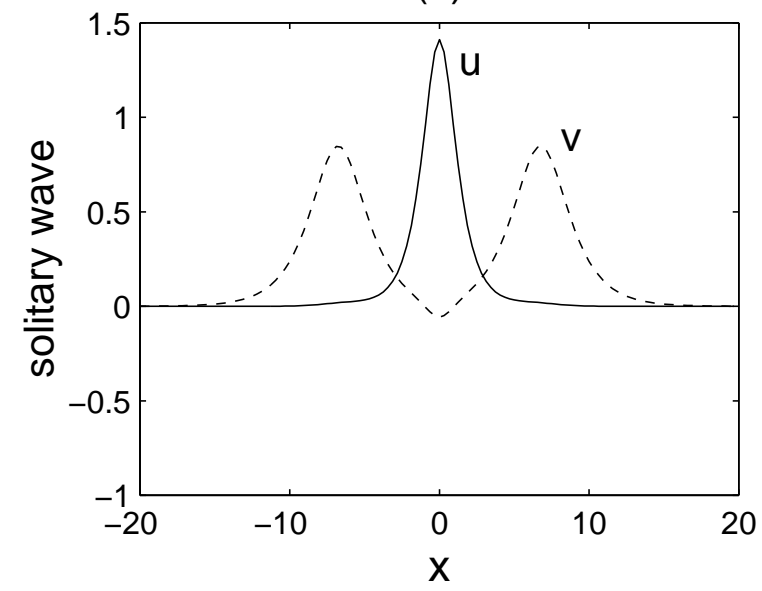

(b)

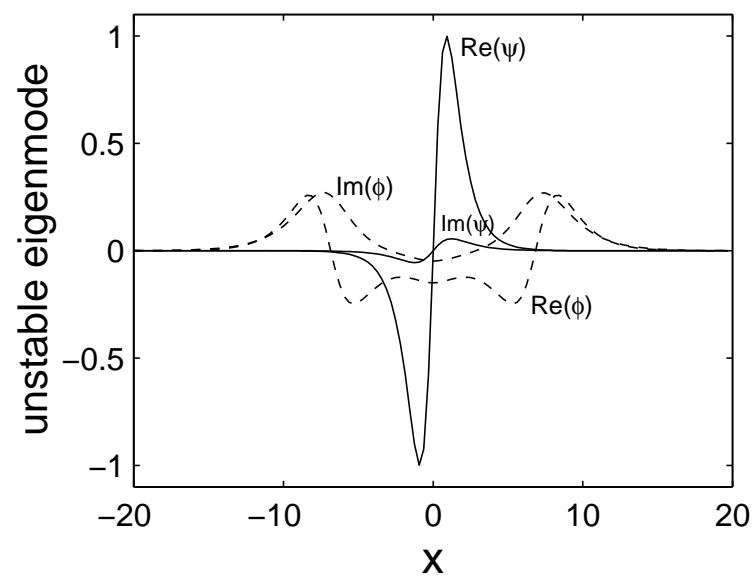

(d)

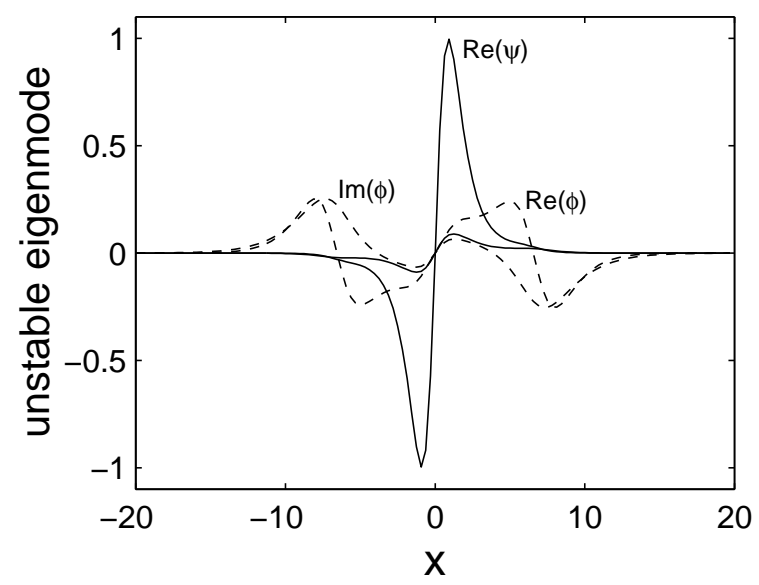

Figure 15: Linear instability of two solitary waves born out of scalar nonlocal bifurcations in the system $(3.1,3.2)$. ( $\mathrm{a}, \mathrm{b})$ the solitary wave and its unstable eigenmode at $(\beta, \omega)=(0.29,0.6) ;(\mathrm{c}, \mathrm{d})$ the solitary wave and its unstable eigenmode at $(\beta, \omega)=(1.6,0.6)$. 
(a)

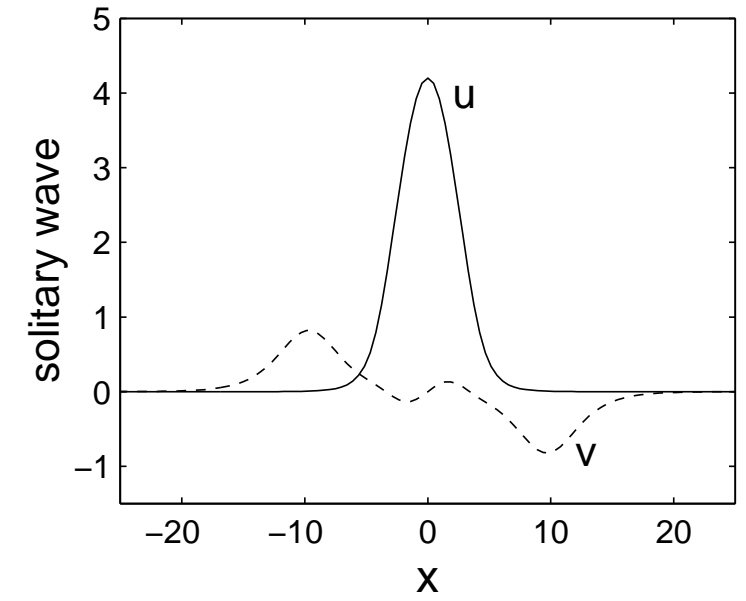

(b)

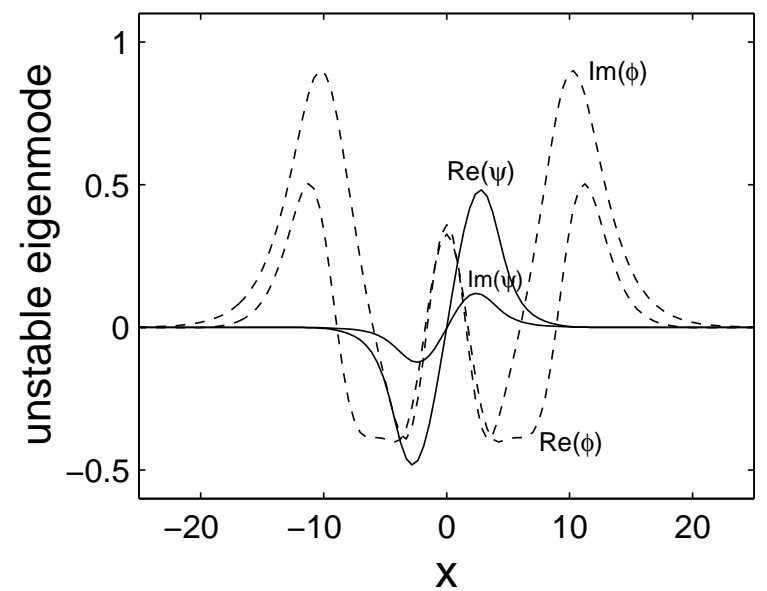

Figure 16: Linear instability of a solitary waves born out of scalar nonlocal bifurcations in the system $(5.1,5.2)$. (a) the solitary wave at $(s, \omega)=(0.81,0.5)$; (b) the unstable eigenmode. 